\title{
Stellar forensics - II. Millisecond pulsar binaries
}

\author{
Brad M. S. Hansen ${ }^{1,2 \star}$ and E. Sterl Phinney ${ }^{2}$ \\ ${ }^{1}$ Canadian Institute for Theoretical Astrophysics, University of Toronto, Toronto, ON M5S 3H8, Canada \\ ${ }^{2}$ Theoretical Astrophysics, 130-33, California Institute of Technology, Pasadena, CA 91125, USA
}

Accepted 1997 October 14. Received 1997 May 30; in original form 1996 November 25

\begin{abstract}
A B S T R A C T
We use the grid of models described in Paper I to analyse those millisecond pulsar binaries whose secondaries have been studied optically. In particular, we find cooling ages for these binary systems that range from $<1$ to $\sim 15$ Gyr. Comparison of cooling ages and characteristic spin-down ages allows us to constrain the initial spin periods and spin-up histories for individual systems, showing that at least some millisecond pulsars had sub-Eddington accretion rates and long magnetic field decay times.
\end{abstract}

Key words: binaries: general - stars: evolution - pulsars: general - pulsars: individual: J0437-4715 - pulsars: individual: J1012+5307 - white dwarfs.

\section{INTRODUCTION}

The ages of millisecond pulsars are important for understanding both their nature and their origin. Ages are usually estimated from the characteristic age $t_{P}=P / 2 \dot{P}$, but such estimates could be seriously in error if the current spin period is still close to the initial spin period at the beginning of the millisecond pulsar phase. If the average millisecond pulsar were significantly younger than its characteristic age, as suggested by Lorimer et al. (1995a), then it would affect current ideas about magnetic field decay in such stars (Kulkarni 1986; Camilo, Thorsett \& Kulkarni 1994b) as well as the possible birth-rate discrepancy between low-mass binary pulsars and low-mass X-ray binaries (Kulkarni \& Narayan 1988; van den Heuvel 1995).

Many millisecond pulsars are found in binaries, often with lowmass degenerate companions. Accurate modelling of the cooling of the companions can allow one to estimate the age of the system (or rather the age of this particular incarnation). Since millisecond pulsars are thought to be spun up as the result of accretion from the companion star, the pulsar will begin to spin down at approximately the same time as the companion shrinks within its Roche lobe, ending mass loss and beginning the process of cooling to its final degenerate white dwarf configuration. Hence the cooling age of the white dwarf should represent the millisecond pulsar age as well.

In Paper I, we calculated accurate cooling models for the lowmass helium core white dwarfs thought to be the companions in these low-mass binary pulsar systems. In this paper we shall now apply the models to the optical observations of these systems to infer the cooling ages for such objects. Section 2 reviews the basic concepts used to infer the age of a pulsar from its spin parameters, as well as simple models of magnetic field decay in such a pulsar. In Section 3 we apply our cooling models to the observational data, and in Section 4 we discuss the implications.

${ }^{\star}$ E-mail: hansen@ cita.utoronto.ca

\section{PULSAR SPIN-DOWN}

For a pulsar with period $P$ and spin-down rate $\dot{P}$ related by $\dot{P} \propto P^{2-n}$, the age is given by

$t=\frac{P}{(n-1) \dot{P}}\left[1-\left(\frac{P_{0}}{P}\right)^{n-1}\right]$,

where $n$ is the braking index and $P_{0}$ is the initial period. For magnetic dipole radiation $n=3$, leading to the familiar expression for the pulsar characteristic age $t_{P}=P / 2 \dot{P}$. For some young pulsars, measurements yield a range for $n$ of 2.0 to 2.8 (Lyne 1996 and references therein). However, $n$ is not known for any millisecond pulsars. Because characteristic ages for millisecond pulsars are of the order of gigayears or higher, there is a very real danger that estimates of the pulsar age based on this quantity will be gross overestimates because of the second factor in equation (1).

The characteristic time is calculated from the spin-down rate $\dot{P}$, which can also be seriously affected by the proper motion of the pulsar (e.g. Camilo et al. 1994b). This is because of the changing Doppler shift (Shklovskii 1970), which produces an additional contribution to the period derivative such that

$\frac{\dot{P}}{P}=\left(\frac{\dot{P}}{P}\right)_{\mathrm{i}}+\frac{V_{\perp}^{2}}{c D}=\left(\frac{\dot{P}}{P}\right)_{\mathrm{i}}+2.432 \times 10^{-21}\left(\frac{\mu}{\mathrm{mas} \mathrm{yr}^{-1}}\right)^{2}\left(\frac{D}{\mathrm{kpc}}\right)$,

where $(\dot{P} / P)_{\mathrm{i}}$ is the intrinsic contribution due to pulsar rotation, $D$ is the distance to the pulsar, $V_{\perp} \propto D \mu$ is the transverse velocity and $\mu$ is the proper motion of the pulsar. Once $\mu$ is measured, we may remove this contribution to obtain the intrinsic characteristic time. Let us define $t_{P}^{*}$ as the characteristic age before the correction and $t_{P}$ as the intrinsic pulsar characteristic age. Thus, with $n=3$, the intrinsic characteristic age is found by

$t_{P}=\frac{t_{P}^{*}}{1-2 V_{\perp}^{2} t_{P}^{*} / c D}$. 
We may then obtain an expression for the ratio of the true age of the pulsar to $t_{P}^{*}$ by

$\frac{t}{t_{P}^{*}}=\left[1-\left(\frac{P_{0}}{P}\right)^{2}\right] /\left(1-\frac{2 V_{\perp}^{2} t_{P}^{*}}{c D}\right)$.

Apart from the initial period $P_{0}$, all the quantities on the right-hand side are measurable in principle. An independent age estimate, such as that from the cooling of a white dwarf, can thus be used to estimate the initial period. If the companion is undetectable then only a lower limit to the age is possible, which in turn translates into an upper limit on $P_{0}$ (as long as $2 V_{\perp}^{2} t_{P}^{*} / c D<1$, which is the case unless the intrinsic period derivative is negative).

There is an additional complication, namely the possibility of magnetic field decay. For magnetic dipole radiation, the spin-down rate is given by

$\dot{P}=\left(\frac{8 \pi^{2} R^{6}}{3 I c^{3}}\right) \frac{B^{2}}{P}$,

where $B$ is the magnetic field strength on the surface of the neutron $\operatorname{star}(B$ is in fact $B \sin \alpha$, where $\alpha$ is the angle between the magnetic dipole axis and the spin axis, and so 'decay' could be the result of field alignment). Thus decay of $B$ will alter the spin-down rate. The possibility of field decay was first advanced by Gunn \& Ostriker (1970) but its occurrence is still not conclusively proven. Narayan \& Ostriker (1990) have argued for an exponential field decay with a decay time of $\sim 10^{7}$ yr. Bhattacharya et al. (1992), on the other hand, find no evidence for field decay. Furthermore, there may be a subdivision between 'normal' pulsars and 'recycled' pulsars (i.e. those spun up through accretion in binaries). Kulkarni (1986), Verbunt, Wijers \& Burm (1990) and Camilo et al. (1994b) have argued that the fields on recycled pulsars do not decay, based on the ubiquitous nature of the field strength $\sim 10^{8-9} \mathrm{G}$ amongst this population of objects and on the presence of cool companions such as the ones we address here.

Thus we shall also consider magnetic field decay of the form $B=B_{0} \exp \left(-t / t_{\mathrm{D}}\right)$, where $t_{\mathrm{D}}$ is the decay time. In this case, the equivalent of equation (4) is

$\frac{t}{t_{\mathrm{D}}}=\frac{1}{2} \ln \left\{1+\frac{2 t_{P}^{*}}{t_{\mathrm{D}}} \frac{\left[1-\left(P_{0} / P\right)^{2}\right]}{\left(1-2 V_{\perp}^{2} t_{P}^{*} / c D\right)}\right\}$.

This introduces a second unknown parameter $t_{\mathrm{D}}$ into the equation. Nevertheless, we can obtain a lower limit to $t_{\mathrm{D}}$ by setting $P_{0}=0$, so that, using equation (6), we obtain

$\frac{t_{\mathrm{D}}}{2 t}\left[\exp \left(2 t / t_{\mathrm{D}}\right)-1\right]<\frac{t_{P}^{*} / t}{\left(1-2 V_{\perp}^{2} t_{P}^{*} / c D\right)}=\frac{t_{p}}{t}$.

Once we have an estimate for $t$, the left-hand side is a monotonically decreasing function of $t_{\mathrm{D}}$ and the right-hand side consists of measurable quantities. Hence we may constrain the exponential field decay time.

\section{RESULTS}

Most low-mass binary pulsars have helium core companions (inferred from their mass, since one requires $\sim 0.5 \mathrm{M}_{\odot}$ to burn helium to carbon in a stellar interior), although three have companions whose masses indicate that they are carbon/oxygen white dwarfs. These measurements come from pulsar timing and are shown in Table 1 for those pulsars discussed here. In order to determine a cooling age, we need optical identifications of the companions. Those systems with detections or upper limits are shown in Table 2. It is these systems that we shall now discuss in turn. We note that, while the following analysis is quantitative, such an exercise is likely to contain small residual systematic uncertainties resulting both from the use of a heterogeneous sample set from a number of independent groups using different instruments and analysis procedures, and from uncertainties in the atmospheric composition and modelling. The cooling curves used in this procedure will be made public to enable improved estimates to be made in the future with better understanding of individual measurement errors and better atmospheric models.

To determine a conservative and robust effective temperature range from the observed colours, we have assumed a blackbody

Table 1. Timing information for optically measured pulsar binaries.

\begin{tabular}{|c|c|c|c|c|c|c|c|c|}
\hline Name & $\begin{array}{c}P \\
(\mathrm{~ms})\end{array}$ & $\begin{array}{c}\dot{P} \\
10^{-20}\end{array}$ & $\begin{array}{c}\mathrm{f}(\mathrm{M}) \\
\left(10^{-3} \mathrm{M}_{\odot}\right)\end{array}$ & $\begin{array}{c}P_{\text {orb }} \\
\text { (days) }\end{array}$ & $\begin{array}{c}\mathrm{D} \\
(\mathrm{kpc})\end{array}$ & $\begin{array}{c}\mu \\
\left(\text { mas. }^{-1} r^{-1}\right)\end{array}$ & $\begin{array}{c}t_{p} \\
(\mathrm{Gyr})\end{array}$ & refs \\
\hline \multicolumn{9}{|c|}{ Helium Cores } \\
\hline J0034-0534 & 1.877 & $0.67(6)$ & 1.260 & 1.589 & 1.0 & $\cdots$ & $4.1-9.3$ & 1 \\
\hline $\mathrm{J} 1713+0747$ & 4.570 & $0.853(2)$ & 7.872 & 67.825 & $1.2(4)$ & $6.4(8)$ & $9.2(4)$ & 2 \\
\hline J0437-4715 & 5.757 & $5.71(1)$ & 1.239 & 5.741 & $0.178(26)$ & $135(4)$ & 4.4-49.1 & 3,4 \\
\hline $\mathrm{J} 1640+2224$ & 3.163 & $0.29(2)$ & 5.889 & 175.461 & 1.2 & $\ldots$ & $>16.2$ & 5 \\
\hline $\mathrm{J} 1012+5307$ & 5.255 & $1.46(8)$ & 0.577 & 0.605 & 0.5 & $\ldots$ & $>5.4$ & 6 \\
\hline B0820+02 & 864.8 & $10390(30)$ & 3.004 & 1232.5 & 1.4 & $<13.6$ & 0.13 & 7 \\
\hline B1855+09 & 5.362 & 1.784 & 5.540 & 12.327 & $1.0(3)$ & $6.16(8)$ & $4.95(5)$ & 8 \\
\hline J0751+1807 & 3.479 & $0.80(8)$ & 0.964 & 0.263 & 2.0 & $\ldots$ & $6.3-12.2$ & 9 \\
\hline $\mathrm{J} 2019+2425$ & 3.935 & $0.702(2)$ & 10.65 & 76.512 & 0.9 & $23(1)$ & $14.7-112$ & 10 \\
\hline \multicolumn{9}{|c|}{ Carbon Cores } \\
\hline $\mathrm{J} 2145-0750$ & 16.05 & $2.9(2)$ & 24.03 & 6.839 & 0.5 & $\cdots$ & $>8.2$ & 1 \\
\hline $\mathrm{J} 1022+1001$ & 16.45 & $4.2(3)$ & 82.79 & 7.805 & 0.6 & $\cdots$ & $>5.8$ & 11 \\
\hline B0655+64 & 195.7 & $69(2)$ & 70.95 & 1.029 & 0.5 & $<46$ & 4.4-146 & 12 \\
\hline
\end{tabular}

References: (1) Bailes et al. (1994); (2) Foster, Wolszczan \& Camilo (1993); (3) Johnston et al. (1993); (4) Bell et al. (1995); (5) Foster et al. (1995); (6) Nicastro et al. (1995); (7) Taylor \& Dewey (1988); (8) Kaspi, Taylor \& Ryba (1994); (9) Lundgren, Zepka \& Cordes (1995); (10) Nice, Taylor \& Fruchter (1993); (11) Camilo (1996); (12) Jones \& Lyne (1988).

All distances without quoted errors are assumed to be subject to a 30 per cent distance error due to the electron density model of Taylor \& Cordes. 
Table 2. Optically identified white dwarf companions to pulsars.

\begin{tabular}{|c|c|c|c|c|c|c|c|}
\hline Name & $\mathrm{E}_{B-V}$ & $\begin{array}{l}T_{\text {eff }} \\
(\mathrm{K})\end{array}$ & $m_{B}$ & $\mathrm{~m}_{V}$ & $\mathrm{~m}_{R}$ & $\mathrm{~m}_{I}$ & refs \\
\hline \multicolumn{8}{|c|}{ Helium Cores } \\
\hline J0034-0534 & 0.00 & $<3500$ & $\cdots$ & $>26.8$ & $>25.0$ & $24.8(3)$ & 1,2 \\
\hline $\mathrm{J} 1713+0747$ & 0.08 & $3430(270)$ & $>27.1$ & $26.0(2)$ & $\ldots$ & $24.1(1)$ & 1 \\
\hline J0437-4715 & 0.07 & $4610(200)$ & $22.19(8)$ & $20.84(2)$ & $20.07(3)$ & $19.51(5)$ & $3,4,5$ \\
\hline $\mathrm{J} 1640+2224$ & 0.05 & 4460(1125) & $\cdots$ & $26.0(3)$ & $24.5(3)$ & $24.6(2)$ & 1,6 \\
\hline $\mathrm{J} 1012+5307$ & 0.00 & $8550(25)^{a}$ & $19.78(4)$ & $19.58(2)$ & $19.49(4)$ & $19.32(4)$ & 7,8 \\
\hline В0820+02 & 0.03 & $15250(1250)$ & .. & $22.8(1)$ & $\ldots$ & $\ldots$ & 9,10 \\
\hline B1855+09 & 0.5 & $\ldots$ & $\cdots$ & $>25.4$ & $>24.6$ & $>23.4$ & 11,12 \\
\hline J0751+1807 & 0.03 & $\ldots$ & $\cdots$ & $>23.5$ & $\cdots$ & $\cdots$ & 8 \\
\hline $\mathrm{J} 2019+2425$ & $>0.2$ & $5400(1300)$ & $\ldots$ & 26.4(4) & $\cdots$ & $25.0(3)$ & 6 \\
\hline \multicolumn{8}{|c|}{ Carbon/Oxygen Cores } \\
\hline $\mathrm{J} 2145-0750$ & 0.03 & $6210(890)$ & $23.89(11)$ & 23.7(1) & $\cdots$ & $22.97(7)$ & 1 \\
\hline $\mathrm{J} 1022+1001$ & 0.00 & $8050(950)$ & .. & $23.10(4)$ & $\cdots$ & $22.665(7)$ & 1 \\
\hline В0655+64 & 0.05 & $7500(1500)$ & $\cdots$ & 22.2 & 22.1 & .. & 13 \\
\hline
\end{tabular}

References: (1) Lundgren et al. (1996b); (2) Bell et al. (1995); (3) Danziger et al. (1993); (4) Bell et al. (1993); (5) Bailyn (1993); (6) Lundgren et al. (1996a); (7) van Kerkwijk et al. (1996); (8) Lorimer et al. (1995a); (9) van Kerkwijk \& Kulkarni (1995); (10) Koester et al. (1992); (11) Kulkarni et al. (1991); (12) Callanan et al. (1989); (13) Kulkarni (1986).

${ }^{a}$ The error is much smaller than the others because this temperature was determined spectroscopically, rather than photometrically.

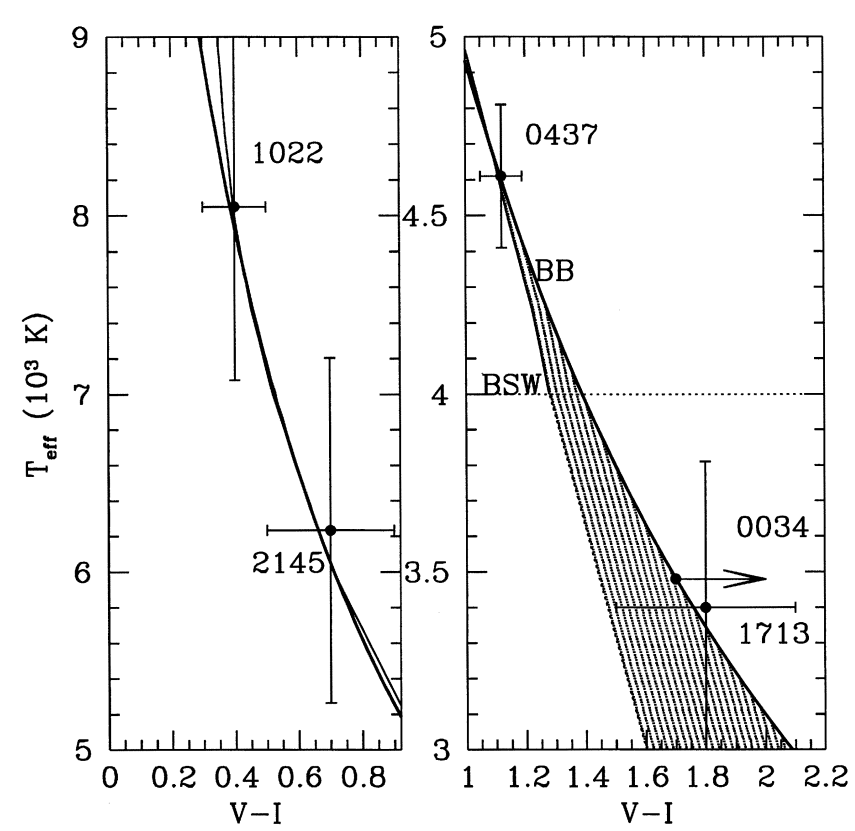

Figure 1. The heavy solid line indicates the blackbody relation (BB) between $T_{\text {eff }}$ and $V-I$. The thin solid curves labelled BSW indicate the colours from the stellar atmosphere calculations of Bergeron et al. (1995) for $\log g=7.5$. The left and right panels show two different colour ranges. In the right panel the shaded area shows the range in which the true colours are expected to lie. Note that we have had to extrapolate the BSW values to temperatures below $4000 \mathrm{~K}$. This turns out to be unimportant, since we are only able to derive upper limits on the temperatures for the two systems in this region anyway. The colours and corresponding temperature estimates are shown for various individual systems by solid circles. Pulsar $\mathrm{J} 1640+2224$ is omitted because the large error bars span both panels, but the temperature inference procedure is the same.

spectrum. We use the bandpasses as described in Bessell (1979). In the case of the more massive white dwarfs, we use the pure hydrogen broad-band colours of Bergeron, Saumon \& Wesemael (1995) (their calculations do not extend to low enough log $g$ ). Fig. 1 shows the blackbody relation between temperature and $V-I$ colour (the most common colour amongst this sample). For $V-I<1.2 / T_{\text {eff }}>4500 \mathrm{~K}$, the blackbody colour is in good agreement with the Bergeron et al. colours. At the lower temperature end, the blackbody approximation is expected to fail because of the contribution of collisionally induced absorption in the hydrogen atmospheres. In Fig. 1 we use the $\log g=7.5$ curves to give us a limit on the deviation of our blackbody colours from the true colours. We do this because the collisionally induced absorption is an inherently high-density phenomenon (since it requires collisional interactions) and thus the deviations from a blackbody should be less for lower gravities (which have lower photospheric pressures). Thus by using this procedure we believe that our temperature estimates are robust.

In the following sections, we shall derive cooling ages for the various binary systems, using the cooling models described in Paper I. When the companion is thought to be a carbon/oxygen core white dwarf as opposed to a helium core white dwarf, we use the latest models derived using the model of Wood (1992). We shall also convert these results into constraints on the initial periods of the millisecond pulsars. In order to obtain conservative estimates, we consider dispersion measure distances to be accurate to within 30 per cent, transverse velocities up to $100 \mathrm{~km} \mathrm{~s}^{-1}$ for systems without a measured proper motion, ${ }^{1}$ and a braking index between $n=2$ and 3 .

\subsection{PSR J0437-4715}

We shall start with the best-determined case. The PSR J0437-4715 system contains the closest known millisecond pulsar, at a nominal dispersion measure distance of $0.14 \mathrm{kpc}$, with a 30 per cent error (Taylor \& Cordes 1993). Bell et al. (1995) report a lower limit of the parallax distance (from pulsar timing) of $90 \mathrm{pc}$. Sandhu et al. (1997), using improved dispersion measurements, report a timing parallax distance of $0.178 \pm 0.026 \mathrm{kpc}$. VLBI parallax

\footnotetext{
${ }^{1}$ Millisecond pulsar velocities are lower than the average velocities of younger pulsars (Lyne \& Lorimer 1994; Hansen \& Phinney 1997).
} 


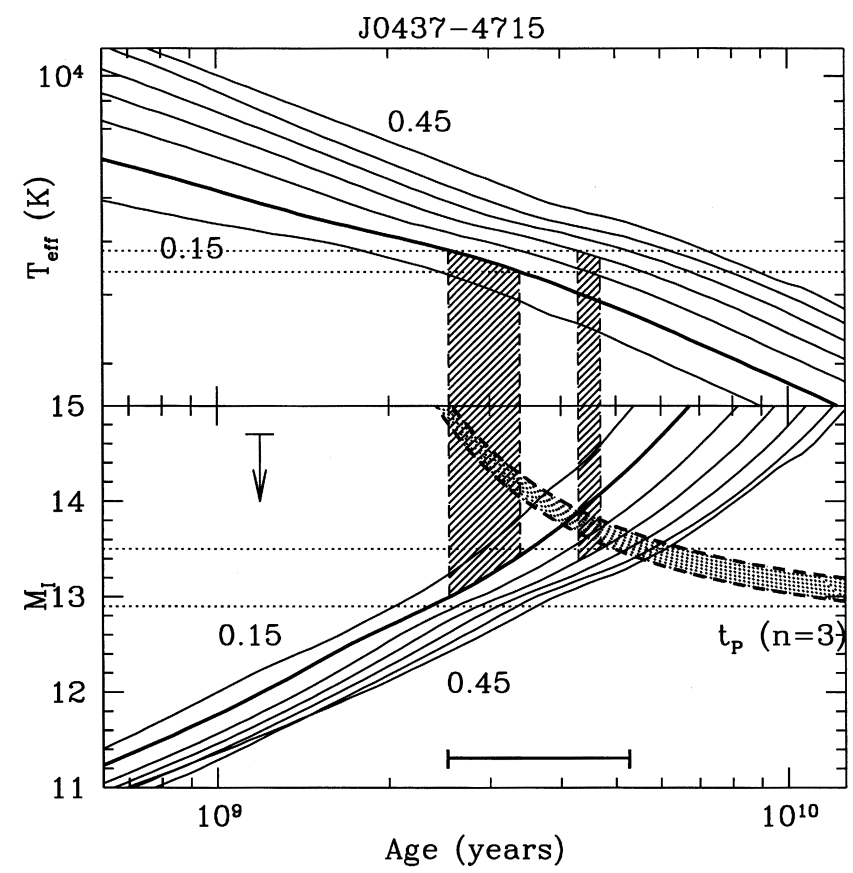

Figure 2. J0437-4715: we show the effective temperature and absolute $I$ magnitude as a function of age for cooling sequences of mass 0.15 to 0.45 $\mathrm{M}_{\odot}$, in steps of $0.05 \mathrm{M}_{\odot}$. The heavy solid line is the $0.20-\mathrm{M}_{\odot}$ model. The horizontal dotted lines in the upper panel indicate the allowed range of $T_{\text {eff }}$. In the lower panel, the dotted lines indicate the allowed range in absolute magnitude from the observations using the timing parallax distance (the upper limit of the absolute magnitude due to the Bell et al. measurement is also shown). In order for a solution to be consistent, it must satisfy both observational criteria. The vertical shaded regions indicate two consistent solutions. Given the apparent $I$ magnitude, each absolute magnitude corresponds to a different distance, and hence a different characteristic age (once corrected for the Shklovskii term). This is shown by the heavy dashed lines and the lightly shaded region, corresponding to a spin-down index of $n=3$. The width of this region is dominated by the uncertainty in the proper motion. The models shown here are for the 'thick' hydrogen layer models (as defined in Paper I). The bar at the bottom indicates the allowed range of cooling ages.

measurements are also expected, which will eventually determine the most accurate distance to this pulsar. PSR J0437-4715 has a

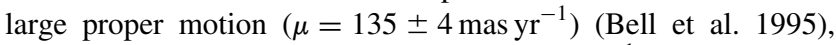
which gives it a transverse velocity of $115 \mathrm{~km} \mathrm{~s}^{-1}$ at $180 \mathrm{pc}$. This affects the spin-down rate, leading to a kinematically corrected characteristic age of 4.5-50.3 Gyr, depending on the distance to the pulsar based on the parallax.

Optical observations of the companion have been carried out by Bell, Bailes \& Bessell (1993), Bailyn (1993) and Danziger, Baade $\&$ Della Valle (1993). Between them these three groups measured $B, V, R$ and $I$ magnitudes. The best data are from Danziger et al. and we will use their measurements. Using the $V-I$ colour and our blackbody approximation, we obtain a temperature estimate of $T_{\text {eff }}=4600 \pm 200 \mathrm{~K}$.

Given the above parallax distance, we can find the absolute magnitude range allowed, namely $M_{I}=13.2 \pm 0.3$. Our models must satisfy both the temperature and magnitude requirements. We can find consistent solutions for all mass $0.15-0.375 \mathrm{M}_{\odot}$ models as shown in Fig. 2. (If we use only the lower distance limit of Bell et al. then solutions for all masses are possible.) These models are for a hydrogen envelope of $3 \times 10^{-4} \mathrm{M}_{\odot}$ (thick hydrogen envelope in the

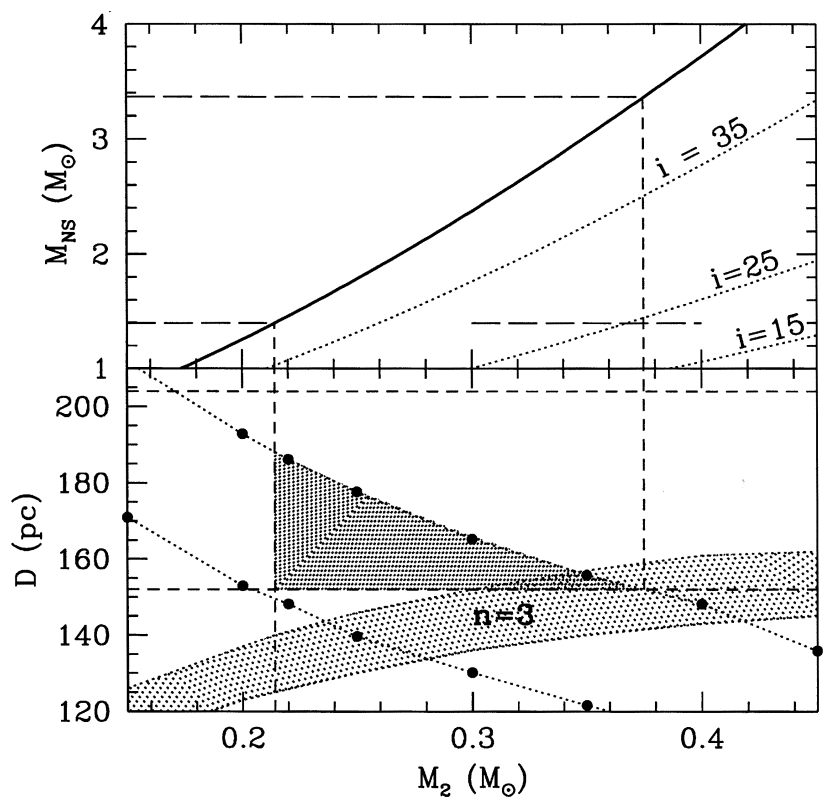

Figure 3. The upper panel demonstrates the constraints on the relationship between neutron star and companion mass. The heavy solid line is the upper limit on $M_{\mathrm{NS}}$ obtained from the mass function and the Sandhu et al. limit on the inclination. The dotted lines indicate the same relation for other allowed inclinations. The solid points in the lower panel span the distance range allowed for each white dwarf mass given the temperature constraints. The horizontal dashed lines are the timing parallax distance limits and the vertical dashed line at $0.214 \mathrm{M}_{\odot}$ is obtained from the requirement that $M_{\mathrm{NS}}>1.4 \mathrm{M}_{\odot}$. Thus the heavy shaded region is that range of distance and companion mass allowed by all contraints. The lightly shaded region covers the range in which the $n=3$ timing age is expected to lie (the uncertainty in the proper motion is responsible for the width of this region).

terminology of Paper I). Similar analysis with thin hydrogen envelope models $\left(10^{-6} \mathrm{M}_{\odot}\right)$ yields similar answers, with cooling ages $\sim 0.3$ Gyr smaller on average.

Further timing measurements by Sandhu et al. have detected, in addition to the parallax, a rate of change in the projected orbital separation $x=a \sin i$, which they interpret as a change in the inclination angle. Assuming that this is the result of the known proper motion, this implies an upper limit to the inclination angle $\left(i<43^{\circ}\right)$ and a new lower limit to the mass of the companion, $M \sim 0.22 \mathrm{M}_{\odot}$. This constrains the mass range to lie between 0.22 and $0.375 \mathrm{M}_{\odot}$ (for a $1.4-\mathrm{M}_{\odot}$ neutron star) and the cooling age is thus 2.5-5.3 Gyr. If we reverse the argument, we can use the upper limit on the companion mass to constrain the neutron star mass to be $<3.36 \mathrm{M}_{\odot}{ }^{2}{ }^{2}$ Furthermore, as we can see from Fig. 3, this further restricts the distance to the system. Using the companion mass limit, the allowed cooling models and the apparent magnitude, one can restrict the distance to $<188 \mathrm{pc}$ (which implies that the intrinsic period derivative is $\dot{P}>6.16 \times 10^{-21}$ ). This allows us to set a better upper limit on the timing age of 14.9 Gyr. If we assume a pulsar mass of $1.4 \mathrm{M}_{\odot}$, then the upper companion mass limit also gives us a lower limit on the inclination $i>24^{\circ}$. These various constraints are demonstrated in Fig. 3, which illustrates the relationships between the various constraints on distance and mass.

${ }^{2}$ Our results differ from those of Sandhu et al. in the interpretation of the optical measurements. They used results based on an average of the various different measurements, while we use only what we consider to be the best measurement, i.e. that of Danziger et al. 


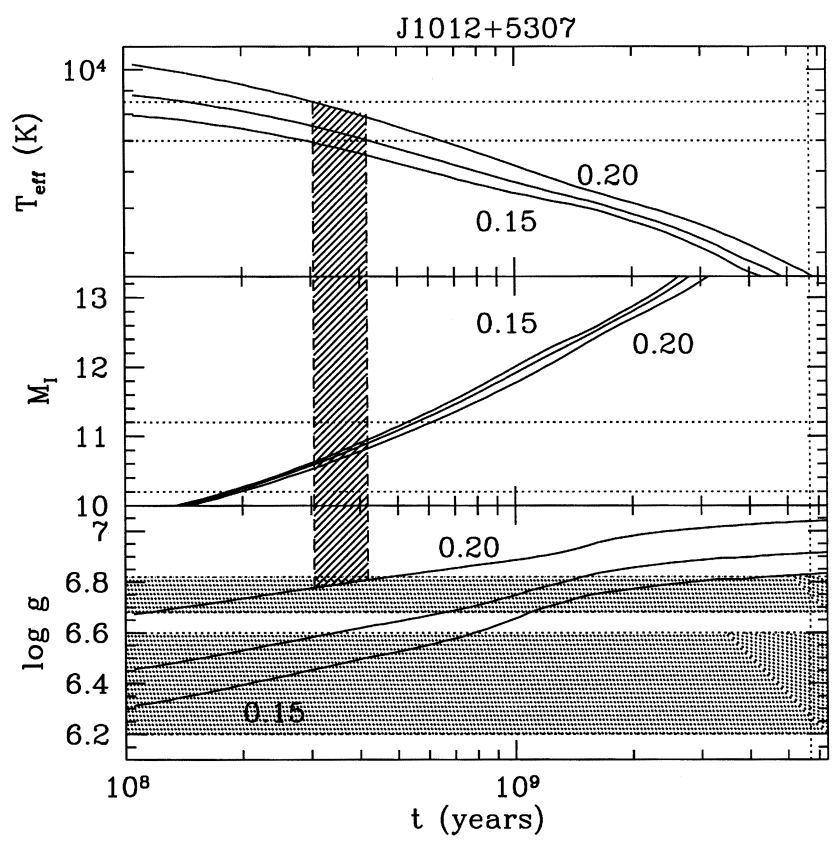

Figure 4. J1012+5307: here we use the effective temperature, luminosity and gravity to constrain the age and mass of the white dwarf companion. The solid lines are models with a thick hydrogen atmosphere and masses of 0.15 , 0.17 and $0.20 \mathrm{M}_{\odot}$ respectively. The two horizontal shaded regions are the gravity values inferred by van Kerwijk et al. (1996) and Callanan \& Koester (1998). The vertical shaded region shows a consistent solution for the $0.2-\mathrm{M}_{\odot}$ thick $\mathrm{H}$ model and the van Kerkwijk et al. gravity, which yields an age significantly lower than the spin-down age, shown by the vertical dotted line at the far right. The Callanan \& Koester gravity will provide a consistent solution for the $0.15-\mathrm{M}_{\odot}$ model.

If we assume that we know the braking index of the pulsar (the default assumption is $n=3$, the value for magnetic dipole radiation), then we may compare the lowest allowed cooling age with the largest characteristic age to get an upper limit on the initial spin period. For pulsar J0437-4715, the proximity and large proper motion mean that the Shklovskii correction is very important for this comparison. For each possible value of the distance, this distance-dependent term yields a value for the characteristic age. Similarly, each different distance leads to a different inferred absolute magnitude. Thus, eliminating the distance, $t_{P}$ may be plotted as a function of $M_{I}$ in Fig. 2. Using the distance and cooling limits derived above, we obtain an upper limit on the initial period of $P_{0}<0.91 P$ or $P_{0}<5.3 \mathrm{~ms}$ for a braking index $n=3$. If we use $n=2$, we may constrain the initial period to lie in the range $2.4<P_{0}<5.3 \mathrm{~ms}$ (the lower limit on the timing age is now larger than the upper limit on the cooling age, thereby allowing us to infer a lower limit for the initial spin period).

For completeness we have also compared the observations with the carbon/oxygen models of Wood (1992). For J0437-4715, we find no $\mathrm{C} / \mathrm{O}$ models that are consistent with the observations. If one lessens the distance restrictions and uses the dispersion measure distance, then one finds that consistent solutions may be obtained for $\mathrm{C} / \mathrm{O}$ models with a mass fraction $\sim 10^{-4}$ of surface hydrogen and $M=0.5-0.55 \mathrm{M}_{\odot}$. The cooling ages allowed by this are 5.6$5.9 \mathrm{Gyr}$, although again it requires a braking index $\sim 2$.

\subsection{PSR J1012+5307}

Even without the benefit of a detailed model, the companion of this system is obviously much brighter than it should be if the system were truly as old as the pulsar characteristic age of $>5.4 \mathrm{Gyr}$ (Lorimer et al. 1995a). The characteristic age could be even larger if the pulsar has a significant transverse velocity.

The system is also closer than average at a distance of $0.52 \mathrm{kpc}$, thus making it an ideal candidate for more detailed study. van Kerkwijk, Bergeron \& Kulkarni (1996) have determined the effective temperature and gravity using the spectroscopic analysis procedure of Bergeron, Wesemael \& Fontaine (1991). The star has $\log g=6.75 \pm 0.07$ and $T_{\text {eff }}=8550 \pm 25 \mathrm{~K}^{3}$ Furthermore, we can use the photometry of Lorimer et al. (1995a) to infer an absolute magnitude. Combining these three data in Fig. 4 we find that we can obtain consistent solutions for both thick and thin hydrogen atmospheres.

Using our mass-radius relations from Paper I, we can determine that the mass limits on the companion are $0.157-0.215 \mathrm{M}_{\odot}$ for a thick hydrogen envelope and $0.13-0.183 \mathrm{M}_{\odot}$ for a thin hydrogen envelope. The corresponding cooling ages are thus $0.18-0.4$ and $\leq 0.23$ Gyr respectively. Thus our combined result for the PSR $\mathrm{J} 1012+5307$ companion is that $M \sim 0.165-0.215 \mathrm{M}_{\odot}$ and the age is $<0.4$ Gyr.

van Kerkwijk et al. (1996) have also measured the radial velocity of the companion, making this a double-lined spectroscopic binary. This allows us to calculate the mass ratio of the two components. The original published value was $M_{\mathrm{NS}} / M_{\mathrm{WD}}=13.3 \pm 0.7$, but it has recently been revised to $M_{\mathrm{NS}} / M_{\mathrm{WD}}=10$ (van Kerkwijk, private communication). Thus, given the companion mass range inferred from the spectroscopic gravity, one can deduce the neutron star mass directly. Using the two different gravities inferred for this object, we deduce neutron star masses of $1.3-1.8 \mathrm{M}_{\odot}$ (Callanan \& Koester 1998) or $1.7-2.1 \mathrm{M}_{\odot}$ (van Kerkwijk et al. 1996). The former number is in excellent agreement with the original author's estimates. These estimates are shown in Fig. 5.

Alberts et al. (1996) claim that a $0.185-\mathrm{M}_{\odot}$ white dwarf burning $\sim 2 \times 10^{-3} \mathrm{M}_{\odot}$ of hydrogen can have the correct gravity and effective temperature at a cooling age commensurate with the pulsar's characteristic age (although, as noted above, the characteristic age could increase further). Their solar metallicity model does not undergo shell flashes, and constitutes this decade's installment in the conflict between the calculations of Webbink (1975) and Iben \& Tutukov (1986)! An examination of fig. 1 and table 1 of Iben \& Tutukov (1986) suggests that the $>50-y r$ timesteps used by Alberts et al. may still not be enough to resolve the shell flash behaviour reported by Iben \& Tutukov. The shell flash results in the burning of much of the surface hydrogen, which leads to the difference in surface hydrogen masses that is the source of the different cooling ages. Although Alberts et al. obtain $\log g \sim 6.7$ and $T_{\text {eff }}=8481 \mathrm{~K}$, we find that our code (which we believe to contain more accurate input physics at the low temperatures appropriate to these studies) yields a lower $\log g \sim$ 6.6 for the same parameters at this temperature, too low to be a consistent solution.

One other uncertainty with this measurement is the possible presence of helium in the white dwarf atmosphere. Bergeron et al. (1991) have shown that a small amount of helium in a hydrogendominated atmosphere can mimic the effect of a larger gravity, a result confirmed by Reid's (1996) comparison of spectroscopic gravities and gravitational redshifts for cool white dwarfs. However, when combined with the temperature and luminosity

${ }^{3}$ As yet unpublished results of Callanan \& Koester (1998) imply a different gravity of $\log g=6.4 \pm 0.2$. We will consider both values in our analysis. 


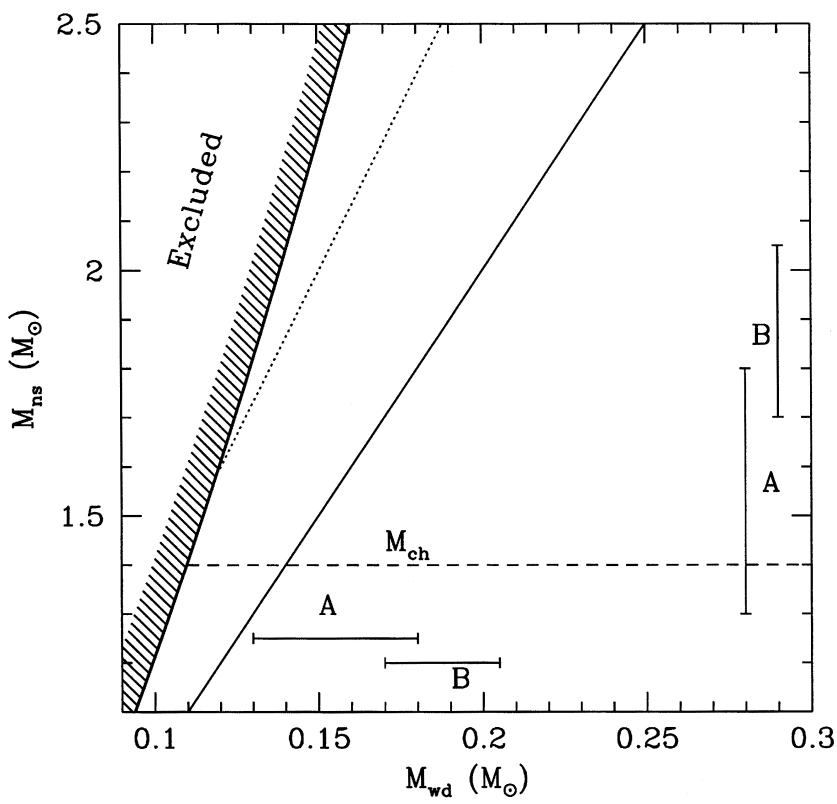

Figure 5. Equation of state constraints from $\mathrm{J} 1012+5307$. The heavy solid line represents the limit from the mass function $f(M)$ measured from the pulsar timing. The diagonal dotted line is the original mass ratio of van Kerkwijk et al. and the solid line the revised value. The two error bars at the bottom indicate the companion masses inferred from the spectroscopic gravity measurements of Callanan \& Koester (A) and van Kerkwijk et al. (B). The corresponding neutron star mass estimates are given by the error bars on the right. The horizontal dashed line indicates the standard Chandrasekhar value for the neutron star mass. It is consistent with the A estimate, although the $\mathrm{B}$ estimate requires a value $\sim 0.3 \mathrm{M}_{\odot}$ larger.

constraints above, our models provide good agreement with pure hydrogen atmospheres. The admixture of trace amounts of helium would not affect the cooling significantly on these time-scales but would measurably raise the gravity. Hence we believe these to be hydrogen-dominated white dwarfs.

With the present uncertain status of the observations, our models yield the following results for the $\mathrm{J} 1012+5307$ system. The companion mass lies in the range $0.13-0.21 \mathrm{M}_{\odot}$, the neutron star mass is in the range $1.3-2.1 \mathrm{M}_{\odot}$, and the age is $<0.6 \mathrm{Gyr}$. Allowing for a braking index of $n=2-3$, this results in the constraint $P_{0}>5.06$ $\mathrm{ms}$. Given the above uncertainties about the gravity measurement, it is useful to calculate the constraints without using the gravity. If we use only the magnitude and temperature, then the white dwarf mass is constrained to lie in the range $0.12-0.32 \mathrm{M}_{\odot}$, and the age is $<0.8$ Gyr. Thus, even ignoring the gravity information, we find that this pulsar is still much younger than the pulsar characteristic age.

\subsection{PSR B0820+02}

The pulsar in this system has the longest spin period $(864.8 \mathrm{~ms})$, largest magnetic field $\left(10^{11.5} \mathrm{G}\right)$ and shortest characteristic time $(0.13$ Gyr) (of course, these are not independent quantities!) of all the lowmass binary pulsars. The companion has been studied optically by Kulkarni (1986) and Koester, Chanmugam \& Reimers (1992). We shall use the Koester et al. apparent magnitude $V=22.76 \pm 0.05$. van Kerkwijk \& Kulkarni (1995) have also identified hydrogen Balmer lines in the companion spectrum, yielding an effective temperature consistent with the photometric temperature estimate of $15250 \pm 1250 \mathrm{~K}$ by Koester et al. The signal-to-noise ratio is not yet good enough to determine a spectroscopic gravity.

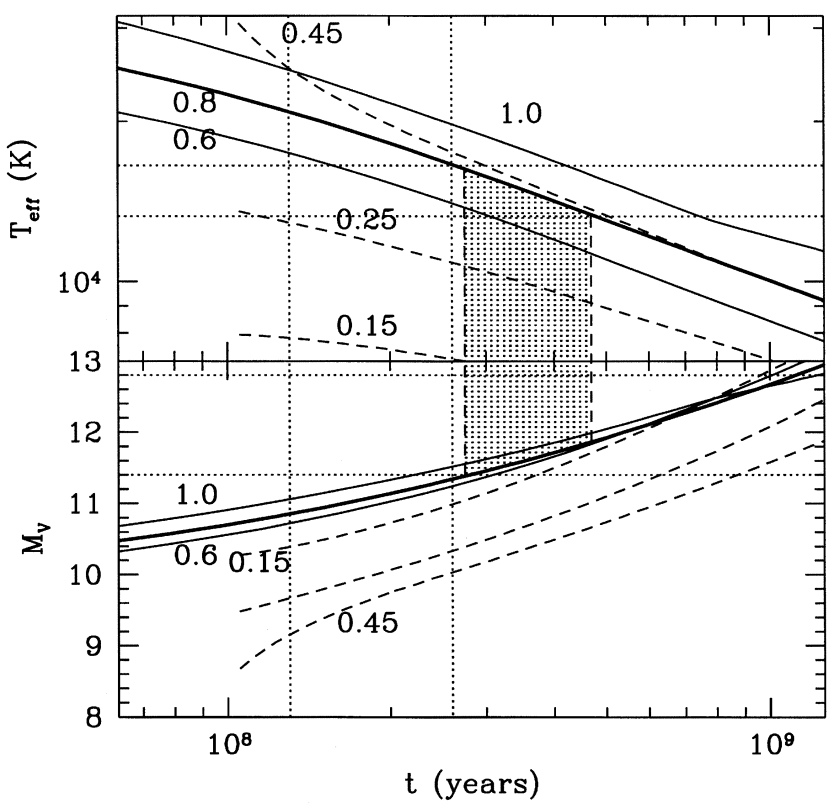

Figure 6. B0820+02: the dashed curves are the models for helium core white dwarfs, while the solid curves are for carbon core white dwarfs. The vertical dotted lines indicates the characteristic age of the pulsar for $n=3$ (lower value) and $n=2$. In the top panel, the horizontal dotted lines indicate the allowed temperature range. In the bottom panel the horizontal dotted lines indicate the absolute magnitude range assuming the dispersion measure distance. The solution for the $0.8-\mathrm{M}_{\odot}$ model is shown as a heavy solid line. The shaded region shows the age range consistent with observations for the $0.8-\mathrm{M}_{\odot}$ model.

The dispersion measure distance to this pulsar is $1.4 \pm 0.4 \mathrm{kpc}$. This yields an absolute $V$ magnitude $M_{V}=12.1 \pm 0.7$. This leads to a problem when we try to find consistent temperature-luminosity solutions for this pulsar. As shown in Fig. 6, the effective temperature and absolute magnitude give a consistent solution for $\mathrm{C} / \mathrm{O}$ models with $M>0.8 \mathrm{M}_{\odot}$. However, these solutions require ages longer than the timing age (even for $n=2$ ). If one requires than $t<t_{P}$, then one is forced to the conclusion that the true distance is larger than the dispersion measure distance. If we use the characteristic age as a constraint (an upper limit to the true age), then we can place some constraints on the acceptable models using only the temperature constraints and infer what the true distance would have to be for a consistent solution.

If we assume a helium core white dwarf (allowing for $n=3$ and $n=2$ ), then the mass is limited to the range $0.27-0.42 \mathrm{M}_{\odot}$, and the true distance has to be $\sim 3.5-4.5 \mathrm{kpc}$ ! If we assume a carbon core white dwarf, the mass range is $0.5-1.0 \mathrm{M}_{\odot}$, with a true distance of $\sim 2-2.8 \mathrm{kpc}$. This is probably the more palatable of the options, which would make this the fourth binary pulsar to contain a 'normal' white dwarf, and the first of those to have not undergone significant spiralling in during its evolution. We note that Koester et al. (1992) also infer a distance range of $1.7-3 \mathrm{kpc}$ based on a similar analysis (although their final published solution is $1.7-1.9 \mathrm{kpc}$, based on the supposed allowed error in the dispersion measure).

If we choose to believe the distance limits, then we are faced with the problem that the white dwarf is older than the pulsar. One possible way of arranging such a situation is if the white dwarf is formed from the initially more massive star (e.g. $\sim 8 \mathrm{M}_{\odot}$ ) which conservatively transfers most of its mass to its initially less massive companion $\left(\sim 4 \mathrm{M}_{\odot}\right)$ thereby creating a system of white dwarf $\left(\sim 0.8 \mathrm{M}_{\odot}\right)$ and massive companion $\left(\sim 11 \mathrm{M}_{\odot}\right)$. The subsequent 
lifetime of this companion is $\sim 2 \times 10^{8} \mathrm{yr}$ (approximately the age difference we infer between white dwarf and pulsar), after which it forms a neutron star. The problem with this scenario is that it will be difficult for the system to remain bound and with small eccentricity (the post-supernova eccentricity is likely to be preserved in a system containing compact stars). Although a suitably directed kick velocity can account for this, the probability of obtaining the correct direction and magnitude $\left(\sim 30 \mathrm{~km} \mathrm{~s}^{-1}\right)$ is rather small. ${ }^{4}$ Nevertheless, if true, this scenario implies a population of isolated pulsars with similar fields and spin-down times with a binary origin.

The determination of a spectroscopic gravity for this star should help to constrain the models, as the helium dwarfs should have $\log g \sim 7$ and the carbon dwarfs should have $\log g \sim 7.8$.

\subsection{PSR J1713+0747}

Lundgren, Foster \& Camilo (1996b) have measured $V=26.0 \pm 0.2$ and $I=24.1 \pm 0.1$ for this companion. This yields a conservative temperature limit of <3800 K. Camilo, Foster \& Wolszczan (1994a) obtained a timing parallax, yielding a distance of $1.2 \pm 0.4 \mathrm{kpc}$. Given this pulsar distance, we use the temperature and the absolute magnitude $M_{I}=13.8 \pm 0.7$. The characteristic age for this pulsar is $9.2 \pm 0.4 \mathrm{Gyr}$, including the correction for proper motion from Camilo et al.

We can find acceptable solutions for all masses from 0.15 to 0.31 $\mathrm{M}_{\odot}$ with a thick $\mathrm{H}$ envelope and for all masses below $0.27 \mathrm{M}_{\odot}$ for a thin $\mathrm{H}$ envelope. However, the mass function for this system restricts the companion mass to be $\geq 0.28 \mathrm{M}_{\odot}$, so that many of these solutions are excluded. If we keep only those models with masses greater than $0.28 \mathrm{M}_{\odot}$, then the cooling age must lie in the range 6.3-6.8 Gyr. We use this result below, but note that, using the dispersion measure distance estimates (which allow smaller distances), we can obtain cooling ages up to $13.2 \mathrm{Gyr}$ and encompassing the full range up to $0.45 \mathrm{M}_{\odot}$. If we assume an $n=3$ braking index, we may constrain the initial period to lie in the range $2.18<P_{0}<2.68 \mathrm{~ms}$ (the lower limit goes to zero for the dispersion measure distances). To be conservative we also consider the effects of $n=2$. This leads to a more conservative upper limit of $3.0 \mathrm{~ms}$.

Inverting the normal procedure for the mass function, we can thus constrain the a neutron star mass to be less than $1.64 \mathrm{M}_{\odot}$ (assuming the parallax distance).

We can find no consistent $\mathrm{C} / \mathrm{O}$ solutions for this object.

\subsection{PSR J1640+2224}

Lundgren et al. (1996b) have also detected the companion in this system, with $V=26.0 \pm 0.3$ and $I=24.6 \pm 0.2$, yielding $T_{\text {eff }}=4460 \pm 1125 \mathrm{~K}$. The characteristic age for this system is at least $16.2 \mathrm{Gyr}$ and the distance $1.2 \mathrm{kpc}$, so that (including distance errors) we have an absolute magnitude $M_{I}=14.2 \pm 0.7$. Once again, the mass function of this system requires the companion to be more massive than $0.252 \mathrm{M}_{\odot}$.

We obtain consistent solutions for all masses $0.25-0.45 \mathrm{M}_{\odot}$. The range of cooling ages obtained is 3.2-12.2 Gyr, including both thick and thin hydrogen envelopes. The extremely low $\dot{P}$ of this pulsar means that $t_{P}$ is both large and sensitive to $V_{\perp}$. Thus we can only obtain a lower limit on the initial period by taking the smallest characteristic age and largest cooling age, yielding $P_{0}>1.6 \mathrm{~ms}$.

${ }^{4}$ In order to maintain small eccentricity, the pre-supernova orbital period has to be similar to the post-supernova period and thus the kick velocity is required to match the change in orbital velocity due to mass loss.

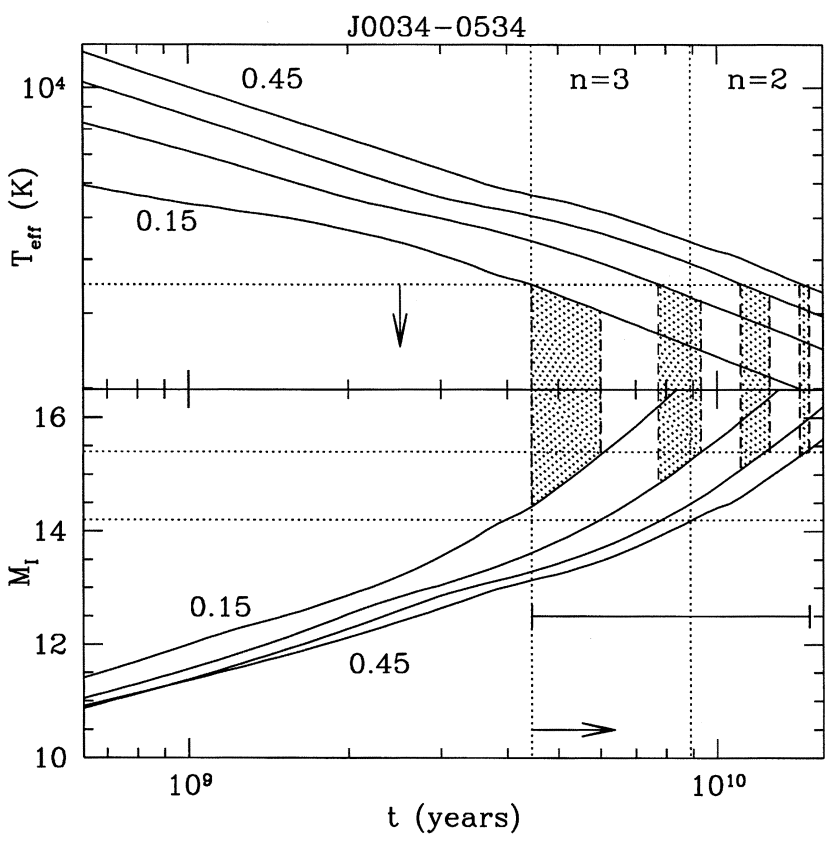

Figure 7. J0034-0534: the shaded regions indicate consistent cooling solutions for $0.15,0.25,0.35$ and $0.45 \mathrm{M}_{\odot}$. The size of the horizontal arrow indicates the change in $t_{P}$ that would result from a transverse velocity of $100 \mathrm{~km} \mathrm{~s}^{-1}$.

\subsection{PSR J0034-0534}

The uncorrected characteristic age is $4.4 \pm 0.4 \mathrm{Gyr}$ and the distance is $1.0 \pm 0.3 \mathrm{kpc}$. The proper motion of this pulsar is not known. However, if the pulsar had a transverse velocity of $100 \mathrm{~km} \mathrm{~s}^{-1}$, the characteristic age could be as much as $9.3 \mathrm{Gyr}$, so we adopt this as an upper limit to the characteristic age. Lundgren et al. (1996b) have detected this companion at $I=24.8 \pm 0.3$ and set a limit of $V>26.8$, which implies that the temperature is $<3500 \mathrm{~K}$. This is sufficient to constrain the ages of cooling models of various masses (see Fig. 7) to lie within the range 4.4-14.9 Gyr. However, if we require that the cooling age be less than the characteristic age (using our upper limit of $9.3 \mathrm{Gyr}$ ), then the mass range is $0.15-0.32 \mathrm{M}_{\odot}$ (assuming $n=3$ ). If we use $n=2$, then all masses are allowed. Thus our conservative upper limit on the initial period is $P_{0}<1.4$ ms (using $n=2$ and assuming a $100 \mathrm{~km} \mathrm{~s}^{-1}$ transverse velocity). A measurement of the proper motion for this pulsar could improve the constraint on the initial period, although it will not prove to be a useful constraint on the nuclear equation of state unless the braking index can be constrained.

\subsection{PSR J2145-0750}

For the carbon/oxygen core white dwarfs, we use Wood's models (Wood 1992), which have a more complete description of crystallization, to perform the same sort of analysis as above. For PSR $\mathrm{J} 2145-0750$, the characteristic age is $>8.2 \mathrm{Gyr}$ and the dispersion measure distance is $0.5 \mathrm{kpc}$. The minimum mass is $0.43 \mathrm{M}_{\odot}$. Optical observations by Lundgren et al. (1996b) give $V=23.7 \pm 0.1$ and $I=23.0 \pm 0.1$. The $V-I$ broad-band colours of Bergeron et al. (1995) yield a temperature estimate of $6235 \pm$ $970 \mathrm{~K}$.

The determination of ages and masses for the carbon sequences requires a little care, because the cores of $M>1 \mathrm{M}_{\odot}$ white dwarfs 


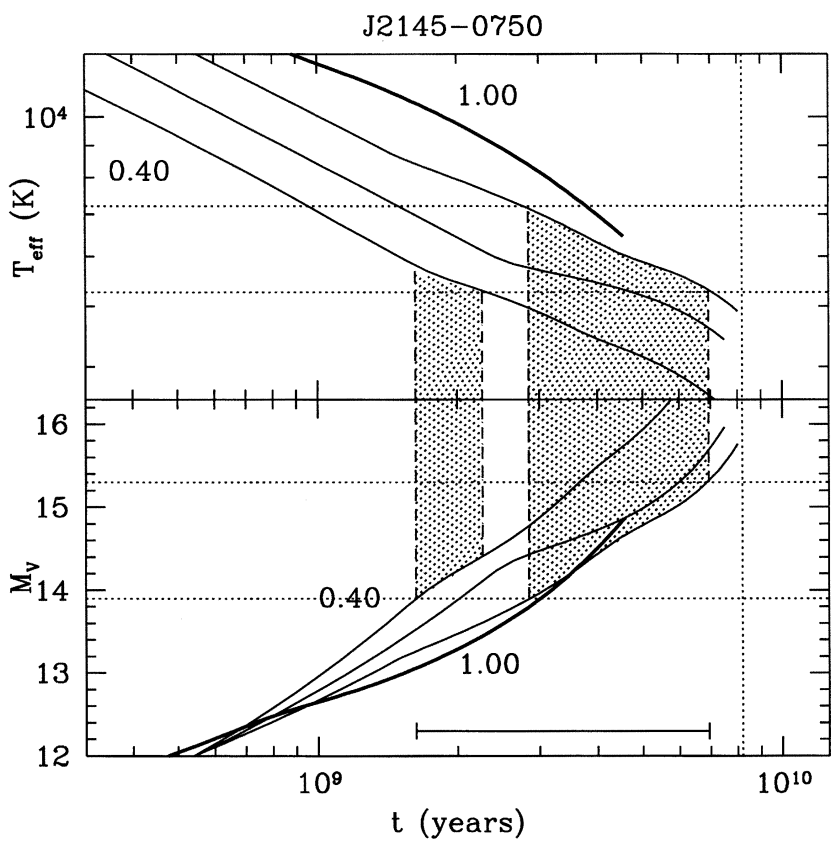

Figure 8. J2145-0750: the solid lines indicate carbon core sequences of mass $0.4,0.7,0.8$ and $1.0 \mathrm{M}_{\odot}$ respectively. The most massive models begin to crystallize after $0.8 \mathrm{Gyr}$, and the least massive after $3.6 \mathrm{Gyr}$. The shaded regions show the consistent solution for the $0.8-\mathrm{M}_{\odot}$ model. The other solutions are omitted because the crossing of the model curves means they lie largely on top of one another.

can begin to crystallize after $\sim 1$ Gyr. This means that the absolute magnitude curves for different masses can cross, making the determination of consistent solutions slightly more complicated. We show the $V$-band magnitudes for this case because the theoretical $M_{V}$ curves are more dispersed than the $M_{I}$ curves. Fig. 8 shows the analysis using the Wood curves for carbon cores, a helium mass fraction of $10^{-2}$ and a hydrogen mass fraction of $10^{-4}$. For models with pure helium envelopes, the age limits are similar. We find consistent models for all masses (in both $\mathrm{C}$ and $\mathrm{O}$ cases, the cores have begun to crystallize for the more massive cases), and a range of cooling ages 1.6-6.9 Gyr.

A transverse velocity of $100 \mathrm{~km} \mathrm{~s}^{-1}$ would change the sign of $\dot{P}$ for this pulsar, so the characteristic age of $8.2 \mathrm{Gyr}$ is only a lower limit. Using the largest cooling age, we can thus get a lower limit on the initial period $P_{0}>6.4 \mathrm{~ms}$ (assuming an $n=3$ braking index).

\subsection{PSR J1022+10}

This pulsar has a spin period of $16.45 \mathrm{~ms}$ and spin-down age $>5.8$ Gyr. It lies at a distance of $0.6 \mathrm{kpc}$ and has been observed by Lundgren et al. (1996b), yielding $V=23.09 \pm 0.04$ and $I=22.665 \pm 0.007$. The mass function for this pulsar requires a companion of mass $M>0.72 \mathrm{M}_{\odot}$, putting this firmly in the carbon/ oxygen mass range. Our temperature estimate for this star is $8050 \pm 950 \mathrm{~K}$. Comparison of the observations with the Wood models yields an age range of 1.5-3.9 Gyr and a mass range $M>0.7 \mathrm{M}_{\odot}$. Thus we obtain $P_{0}>9.4 \mathrm{~ms}$.

\subsection{PSR B0655+64}

This was one of the first pulsars with an optically identified companion. Upper limits on the proper motion mean that we can

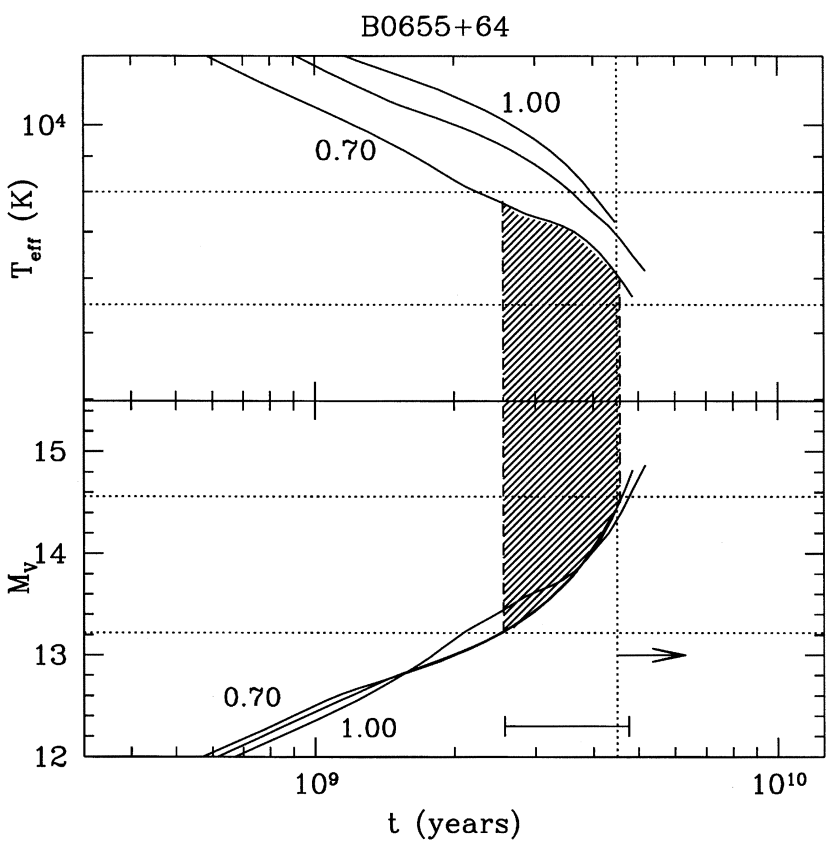

Figure 9. B0655+64: we show here the curves for 0.7, 0.9 and $1.0 \mathrm{M}_{\odot}$ and a helium envelope mass fraction $=10^{-4}$. These models have no hydrogen envelope. The shaded region shows the solution for $0.7 \mathrm{M}_{\odot}$.

constrain the characteristic age to the range 4.4-146(!) Gyr. The distance is $0.48 \mathrm{kpc}$. The mass function constrains the companion to have $M \geq 0.67 \mathrm{M}_{\odot}$. van Kerkwijk \& Kulkarni (1995) have identified the white dwarf companion as a DQ star, i.e. it shows traces of molecular carbon in the optical spectrum. This makes accurate temperature determination difficult but does constrain the temperature to be in the range $5500-8000 \mathrm{~K}$, when the convection zone is deep enough to dredge up trace amounts of carbon from the core and deposit them in the atmosphere (Pelletier et al. 1986). Kulkarni (1986) has measured $V=22.2$ for this star. Since molecular carbon is seen, the helium and hydrogen envelopes must be quite thin. We use the pure helium envelope/carbon core models $\left(q_{\mathrm{He}}=10^{-4}\right)$ of Wood (1992) to calculate the cooling ages in Fig. 9. We find solutions for all models $M \geq 0.7 \mathrm{M}_{\odot}$. The range of cooling ages is $2.5-4.7 \mathrm{Gyr}$. The large range in characteristic age means that no useful limits can be obtained for $n=3$. For $n=2$, the smallest characteristic age and largest cooling age yield $P_{0}>94 \mathrm{~ms}$ (since, in this case, the maximum cooling age is less than the minimum timing age).

\subsection{PSR 2019+2425}

This pulsar has one of the largest characteristic ages (corrected for proper motion) of any pulsar, ranging from 14.7 to $112 \mathrm{Gyr}$, depending on the rather uncertain distance. It lies close to the Galactic plane, which means that extinction is an important problem. Lundgren et al. (1996b) have detected the pulsar companion at $V=26.4 \pm 0.4$ and $I=25.0 \pm 0.3$. To estimate the extinction to the companion, we find the extinction in the general direction of J2019+2425 from Neckel \& Klare (1980). For distances less than 1 $\mathrm{kpc}, A_{V}$ rises linearly to $0.4 \mathrm{mag}$, but then rises steeply to $\sim 3$ mag at a distance of $1.5 \mathrm{kpc}$ (i.e. $1.5 \mathrm{mag}$ for $A_{I}$ ). Using this extinction law and the 30 per cent distance uncertainty, we find that $V-I=1.0 \pm 0.4$, which implies $T_{\text {eff }} \sim 4000-6500 \mathrm{~K}$. Similarly, we estimate the absolute $I$-band magnitude as $M_{I}=14.9 \pm 1.0$. A 


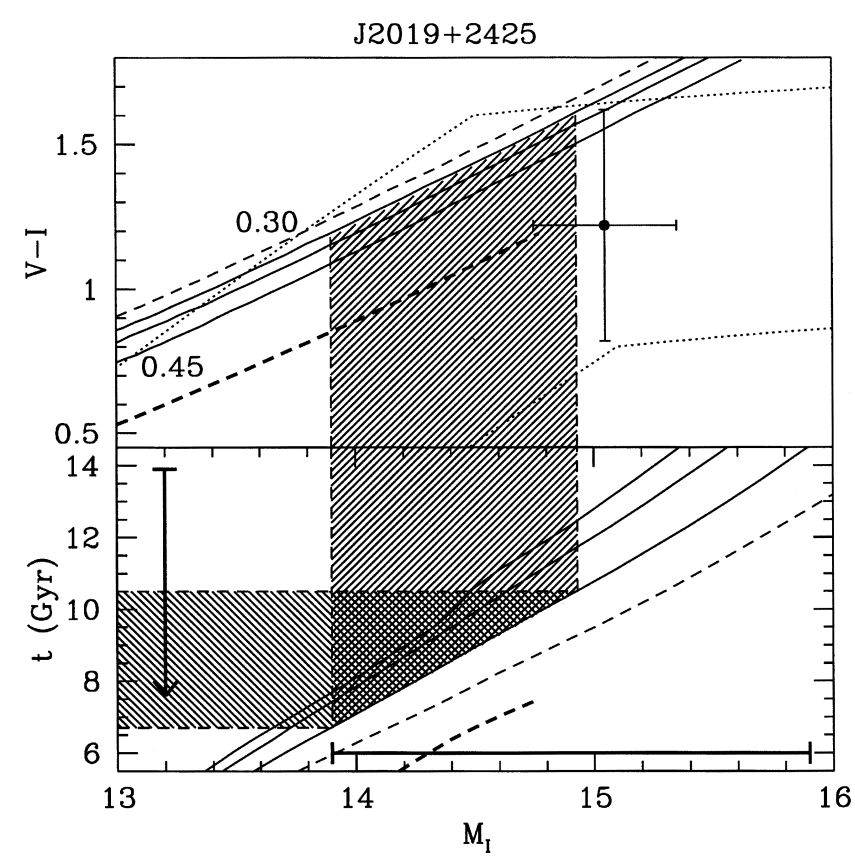

Figure 10. J2019+2425: the solid lines are the models for a $0.35-\mathrm{M}_{\odot}$ thick layer model. The heavy dashed line is for a $0.60-\mathrm{M}_{\odot}$ carbon core model. The dotted line in the upper panel indicates the allowed range of $V-I$ for each $M_{I}$ (and hence each distance). The shaded region demonstrates the consistent solution for the $0.35-\mathrm{M}_{\odot}$ model. The filled circle (with error bars) is the allowed range at the nominal dispersion measure distance. In the bottom panel the vertical and horizontal bars indicate the allowed range of cooling ages (for all models) and the expected range in $M_{I}$ consistent with the dispersion measure uncertainty.

further constraint is that the mass function requires that the companion mass be $>0.32 \mathrm{M}_{\odot}$.

Using these limits we find consistent solutions for all masses above the minimum allowed by the mass function. Fig. 10 shows the solution for the $0.35-\mathrm{M}_{\odot}$ case. The approach taken here is slightly different because the allowed ranges of $T_{\text {eff }}$ and $M_{I}$ are correlated. The large extinction correction implies that the allowed $V-I$ and $M_{I}$ ranges are a function of distance, as shown in Fig. 10. Using this approach we find a range of cooling ages from 7.6 to $13.9 \mathrm{Gyr}$. Although Wood's models again do not extend to low enough temperatures in most cases, modest extrapolation indicates that consistent solutions may also be found with $\mathrm{C} / \mathrm{O}$ models for all masses (a sample 0.6- $\mathrm{M}_{\odot}$ model is shown in Fig. 10). This extends the allowed range to $3.4-13.9 \mathrm{Gyr}$.

Using these values and the range 14.7-112 Gyr for the characteristic age, we may derive a lower limit to the initial period of 0.9 $\mathrm{ms}$ for $n=3$ (or $2.1 \mathrm{~ms}$ for $n=2$ ). Despite the uncertainty in the cooling age resulting from the extinction correction, we find that this result is quite robust, primarily because the measured proper motion constrains the characteristic age to be significantly greater than the age of the Galaxy.

\subsection{PSRs B1855+09 and J0751+1807}

For those systems where we have only upper limits on the companion magnitudes, it is sometimes still possible to place some constraints on the ages and masses of the stars involved.

For PSR $1855+09$, the almost $90^{\circ}$ inclination of this orbit has allowed Kaspi, Taylor \& Ryba (1994) to determine the masses of both components as well as the parallax and distance. The

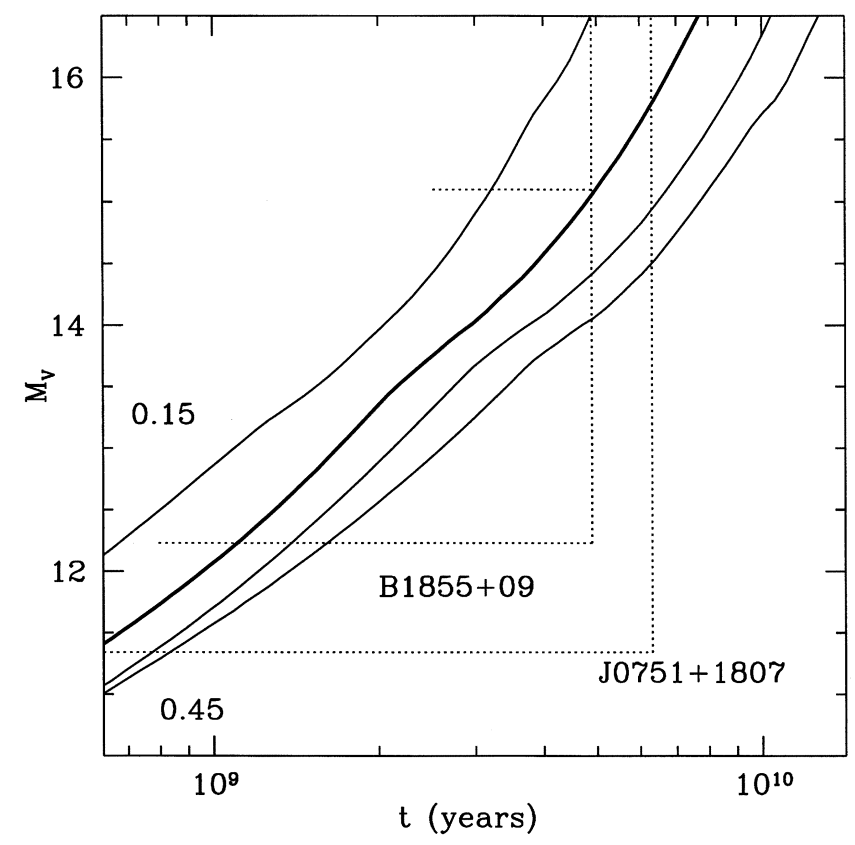

Figure 11. Limits for other pulsar companions. We compare the absolute $V$ magnitudes of our models with the limits determined for two other binary pulsar systems. The vertical dotted lines indicate the characteristic ages for each binary system and the horizontal dotted lines are the magnitude limits determined from the observations. The models are for $0.15,0.25,0.35$ and $0.45 \mathrm{M}_{\odot}$ respectively. The heavy solid line indicates the $0.25-\mathrm{M}_{\odot}$ model (for comparison with the B1855+09 limits).

companion mass is $0.258_{-0.016}^{+0.028} \mathrm{M}_{\odot}$ and the distance is $0.9_{-0.2}^{+0.4}$ $\mathrm{kpc}$. The characteristic age, corrected for kinematic effects, is $4.95 \pm 0.05 \times 10^{9} \mathrm{yr}$. We can thus restrict our model comparisons to the $0.25-\mathrm{M}_{\odot}$ model in this case. Kulkarni, Djorgovski \& Klemola (1991) obtained limits of $R>24.6$ and $I>23.4$, while Callanan et al. (1989) obtained $V>25.4$. Callanan et al. estimated the extinction to the system to be $A_{V}=1.5-2.0 \mathrm{mag} \mathrm{kpc}^{-1}$. Using the extinction law of Savage \& Mathis (1979), this becomes $A_{R}=1.1-1.5$ and $A_{I}=0.7-1.0 \mathrm{mag} \mathrm{kpc}{ }^{-1}$. Fig. 11 shows the comparison of the model curves with each constraint. We use two constraints, using the largest extinctions and furthest distance estimate, and the smallest extinctions and closest distance estimates, to examine the range of possible solutions. We see that the $0.25-\mathrm{M}_{\odot}$ sequence is perfectly consistent with the bounds on the absolute magnitude for all ages from the characteristic age of $4.95 \mathrm{Gyr}$ to a minimum age of $1.4 \mathrm{Gyr}$. This yields a constraint $P_{0}>4.5 \mathrm{~ms}$.

Also shown in Fig. 11 are the detection limits for the companion to J0751+1807, with a characteristic age of 6.3-12.2 Gyr. The only meaningful limit that can be placed on this system is that the cooling time is longer than 0.8 Gyr. So this could still be a slightly older version of the $\mathrm{J} 1012+5307$ system. Given the small orbital period, we might expect a low companion mass $\left(\sim 0.15 \mathrm{M}_{\odot}\right)$, so that extending the detection limit to $V \sim 26$ would detect the companion if it were younger than 2 Gyr. Such a detection would be extremely interesting.

The various constraints on the cooling ages are collected together in Fig. 12.

\section{DISCUSSION}

\subsection{Binary evolution}

The formation of low-mass binary pulsars (LMBPs) has been 


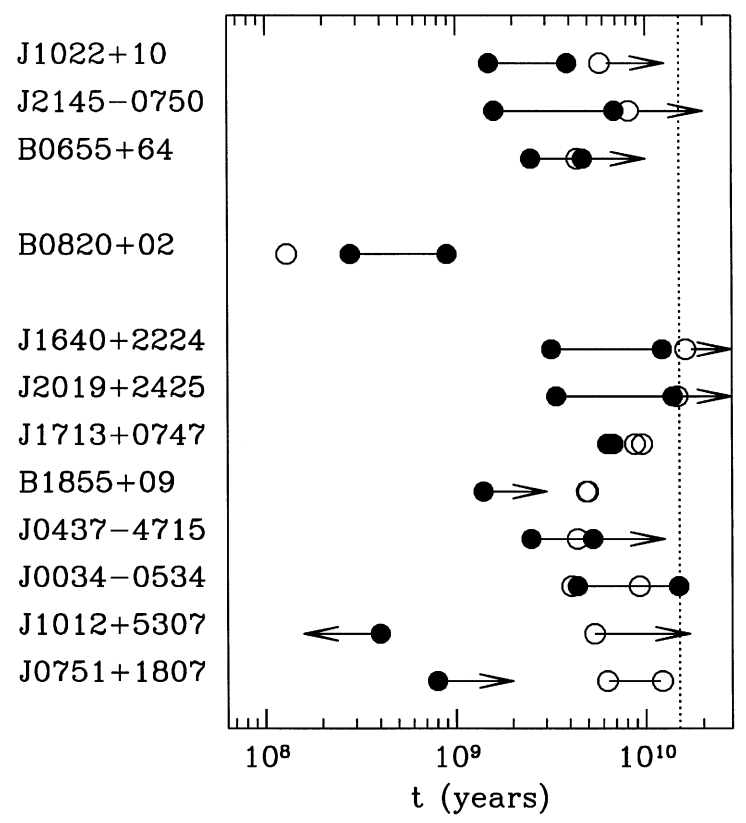

Figure 12. Cooling ages for millisecond pulsar companions. Here we show the constraints on the cooling age for the various binaries discussed in this paper. The open circles indicate characteristic ages (for a braking index $n=3$ ), and the filled circles indicate cooling ages. The uncertainties in the characteristic ages are because of the Shklovskii term. The systems are separated into $\mathrm{C} / \mathrm{O}$ and $\mathrm{He}$ white dwarfs (B0820+02 is placed in limbo due to the uncertainty in its mass) and ordered by increasing orbital period. The dotted vertical line denotes an age of $15 \mathrm{Gyr}$.

discussed by many authors (for reviews see Phinney \& Kulkarni 1994, Verbunt 1993 and references therein). If we consider a binary containing a pulsar and a stellar companion, the binary will undergo mass transfer if the non-degenerate companion begins to expand as a result of nuclear evolution or if the orbit decreases due to magnetic braking or gravitational wave radiation. This mass transfer results in the spin-up of the neutron star to form a millisecond pulsar. The mass loss also means that the companion never evolves far enough to grow a core of mass large enough to ignite helium. Rather, the envelope is lost during the course of the evolution and the remnant of the secondary settles down to a degenerate configuration, a lowmass helium core white dwarf. The fact that giants have a welldefined relationship between core mass and giant radius, allied with the fact that the star must fill its Roche lobe to lose matter to the companion (assuming corotation), means that there exists a relationship between orbital period and secondary mass in the LMBPs (Refsdal \& Weigart 1971; Rappaport et al. 1995; Joss, Rappaport \& Lewis 1987). However, this holds only as long as the secondary star is sufficiently evolved to have a convective envelope when it overflows its Roche lobe. This is because mass loss from the secondary results in expansion of the orbit, shutting off mass loss unless the donor star increases as well (which requires a convective envelope, rather than a radiative one). Thus this scenario describes systems with orbital periods $\sim 50-10^{3} \mathrm{~d}$.

For shorter period systems, the donor star overflows its Roche lobe either on the main sequence or during the transition from the main sequence to the giant branch. The envelope is still primarily radiative in this case, and the star will shrink in response to mass loss. The result is that one needs angular momentum loss mechanisms such as gravitational radiation and magnetic braking to maintain mass transfer in these systems. The competition between

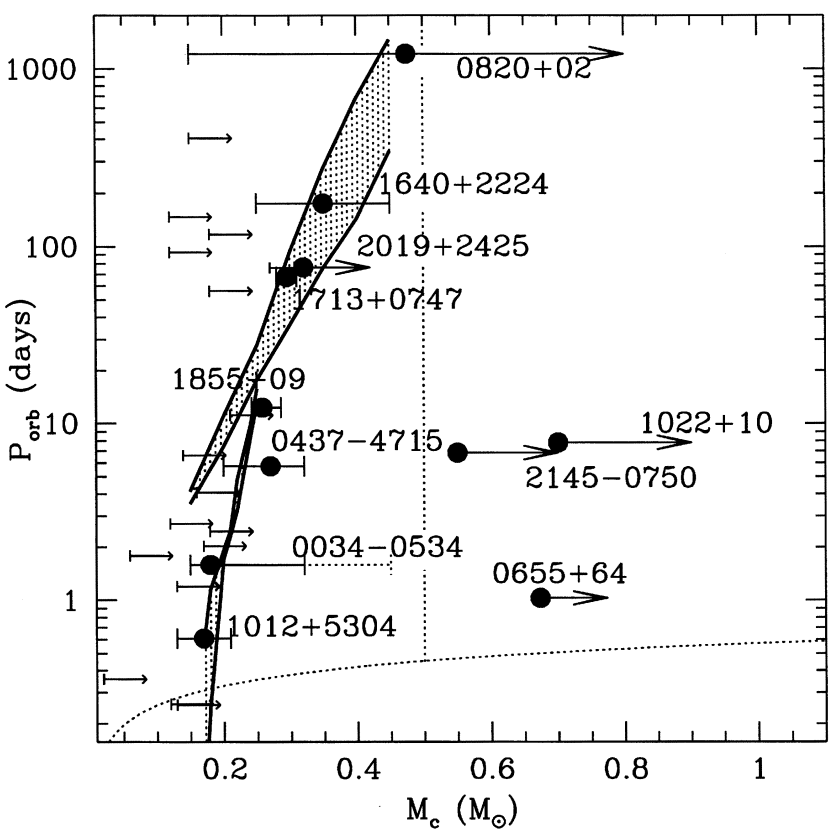

Figure 13. The orbital period-mass relation. The upper shaded region is the parameter space spanned by the models of Rappaport et al. (1993) while the lower shaded region is that spanned by the models of Pylyser \& Savonije (1988). Also shown are the mass constraints for various systems determined in this paper as well as those of Kaspi et al. (1994) for B1855+09 and the mass function limits for those systems without further constraints. The dotted line at the bottom of the diagram indicates the boundary below which the companion will spiral into a $1.4-\mathrm{M}_{\odot}$ neutron star in a Hubble time due to gravitational radiation. The vertical dotted line indicates the dividing line between carbon cores $\left(>0.5 \mathrm{M}_{\odot}\right)$ and helium cores $\left(<0.5 \mathrm{M}_{\odot}\right)$. The error bars for $\mathrm{B} 0820+02$ and $\mathrm{J} 2019+2425$ are from two overlapping mass estimates, one for a helium core white dwarf and one for a carbon core. J0034-0534 has two upper mass limits, for $n=3\left(0.32 \mathrm{M}_{\odot}\right)$ and $n=2$ $\left(0.45 \mathrm{M}_{\odot}\right)$.

these loss mechanisms and the mass transfer induced evolution of the system leads to a very steep relationship between final orbital period and initial orbital period/final core mass (Pylyser \& Savonije 1988; Coté \& Pylyser 1989).

In Fig. 13 we compare our mass determinations for these companions with the results of Rappaport et al. (1993) and Pylyser \& Savonije (1988) (omitting models in the latter sample where the accretor was far from $1 \mathrm{M}_{\odot}$ ). We find excellent agreement with the models, especially that of Pylyser \& Savonije, where the mass estimates of B1855+09, J0034-0534, J0437-4715 and $\mathrm{J} 1012+5307$ trace out the mass-period relation very nicely. Thus the two different scenarios provide a natural explanation for the orbital period gap between 20 and $50 \mathrm{~d}$.

\subsection{Neutron star spin-up}

Under the assumption that the magnetic field does not decay, the cooling age of the white dwarf allows us to estimate the initial spin period of the millisecond pulsar, by inverting formula (1). These estimates are shown in Table 3.

The simplest theories regarding spin-up of recycled pulsars to millisecond periods (Smarr \& Blandford 1976; Ghosh 1995 and references therein) predict that the initial period should be equal to the equilibrium spin period of the neutron star of magnetic field $B$ 
Table 3. Cooling ages and initial spin periods.

\begin{tabular}{lcccccc}
\hline Name & $\begin{array}{c}P \\
(\mathrm{~ms})\end{array}$ & $\begin{array}{c}t_{P} \\
(\mathrm{Gyr})\end{array}$ & $\begin{array}{c}t_{\text {cool }} \\
(\mathrm{Gyr})\end{array}$ & $\begin{array}{c}P_{0}{ }^{a} \\
(\mathrm{~ms})\end{array}$ & $\begin{array}{c}P_{0}{ }^{b} \\
(\mathrm{~ms})\end{array}$ & $\begin{array}{c}t_{\mathrm{D}} \\
\mathrm{Gyr}\end{array}$ \\
\hline Helium Cores \\
$\mathrm{J} 0034-0534$ & 1.88 & $4.1-9.3$ & $4.4-14.9$ & $<1.4$ & $<1.4$ & $>6.5$ \\
$\mathrm{~J} 1713+0747$ & 4.57 & $8.8-9.6$ & $6.3-6.8$ & $2.2-2.7$ & $2.8-3.1$ & $>15.6$ \\
$\mathrm{~J} 0437-4715$ & 5.76 & $4.5-50.3$ & $2.5-5.3$ & $<5.3$ & $2.4-5.3$ & $>1.1$ \\
$\mathrm{~J} 1640+2224$ & 3.16 & $>16.2$ & $3.2-12.2$ & $>1.6$ & $>2.0$ & $<45$ \\
$\mathrm{~J} 1012+5307$ & 5.26 & $>5.4$ & $<0.4$ & $>5.1$ & $>5.1$ & $<0.2$ \\
$\mathrm{~B} 1855+09$ & 5.36 & $4.9-5.0$ & $>1.4$ & $<4.5$ & $<4.6$ & $>1.3$ \\
$\mathrm{~J} 0751+1807$ & 3.48 & $>6.3$ & $>0.8$ & $\cdots$ & $\cdots$ & $>0.4$ \\
$\mathrm{~J} 2019+2425$ & 3.94 & $14.7-112$ & $3.4-14$ & $0.9-3.9$ & $2.1-3.9$ & $>1.3$ \\
& \multicolumn{7}{c}{ Carbon/Oxygen Cores } & & \\
$\mathrm{J} 2145-0250$ & 16.05 & $>8.2$ & $1.6-6.9$ & $>6.4$ & $>9.3$ & $<39.7$ \\
$\mathrm{~J} 1022+1001$ & 16.45 & $>5.8$ & $1.5-3.9$ & $>9.4$ & $>10.9$ & $<10.5$ \\
B0655+64 & 195.7 & $4.4-202$ & $2.5-4.7$ & $\cdots$ & $>91$ & $>0.9$ \\
\hline
\end{tabular}

${ }^{a}$ Based on $n=3$.

${ }^{b}$ Based on $n=2$.

accreting at a rate $\dot{M}$. This predicts an initial spin period

$P_{0}=1.89 \mathrm{~ms} B_{9}^{6 / 7}\left(\frac{\dot{M}}{\dot{M}_{\text {Edd }}}\right)^{-3 / 7}$,

where $\dot{M}_{\text {Edd }}$ is the Eddington accretion rate. Thus a comparison between the inferred initial spin period and magnetic field can determine the accretion rate on to the neutron star during spin-up. However, this inversion is complicated somewhat by the uncertainty in the macroscopic dimensions of the neutron star. Fig. 14 shows the spin period-magnetic field diagram for the millisecond pulsars, scaled in such a way that it reflects observational parameters $(P$ and $\dot{P})$ only. This is done by plotting

$\frac{\mu_{26}^{2}}{I_{45}}=1.026\left(\frac{P}{1 \mathrm{~ms}}\right)\left(\frac{\dot{P}}{10^{-20}}\right)$,

where $\mu=R^{3} B$ is the magnetic moment (in this case expressed in terms of units of $10^{26} \mathrm{G} \mathrm{cm}^{3}$ ) and $I$ is the moment of inertia of the pulsar. Thus we infer accretion rates $\sim 10^{-2}-0.1 \dot{M}_{\text {Edd }}$, possibly even somewhat lower in some cases. A similar conclusion was reached by Lundgren et al. (1996b). This is consistent with the findings that low-mass X-ray binaries have a range of sub-Eddington accretion rates (Bradt \& McClintock 1983).

\subsection{Magnetic field decay}

Although many authors have noted that millisecond pulsars must have very long magnetic field decay times (Kulkarni 1986; Camilo et al. 1994a), the determination of a cooling age allows us to make a quantitative estimate of $t_{\mathrm{D}}$ in the context of the paradigm outlined in Section 2. To get the lower limits on $t_{\mathrm{D}}$ using equation (7), we must use the lower limits on both the distance and the cooling age (and assume a value for $n$; we will use $n=3$ below). For pulsar $\mathrm{J} 1713+0747$ we find a lower limit of $15.6 \mathrm{Gyr}$ for $t_{\mathrm{D}}$ (essentially because the cooling age is restricted to be quite close to the spindown age), which is the largest value amongst all our sample. PSR J0034-0534 also yields a strong constraint (6.5 Gyr) although most of the other systems yield weaker constraints $(\sim 1 \mathrm{Gyr}$ in most cases). These results strongly support the view that millisecond pulsar fields do not decay at all. Fig. 15 shows the limits using equation (7) for the various binary systems.

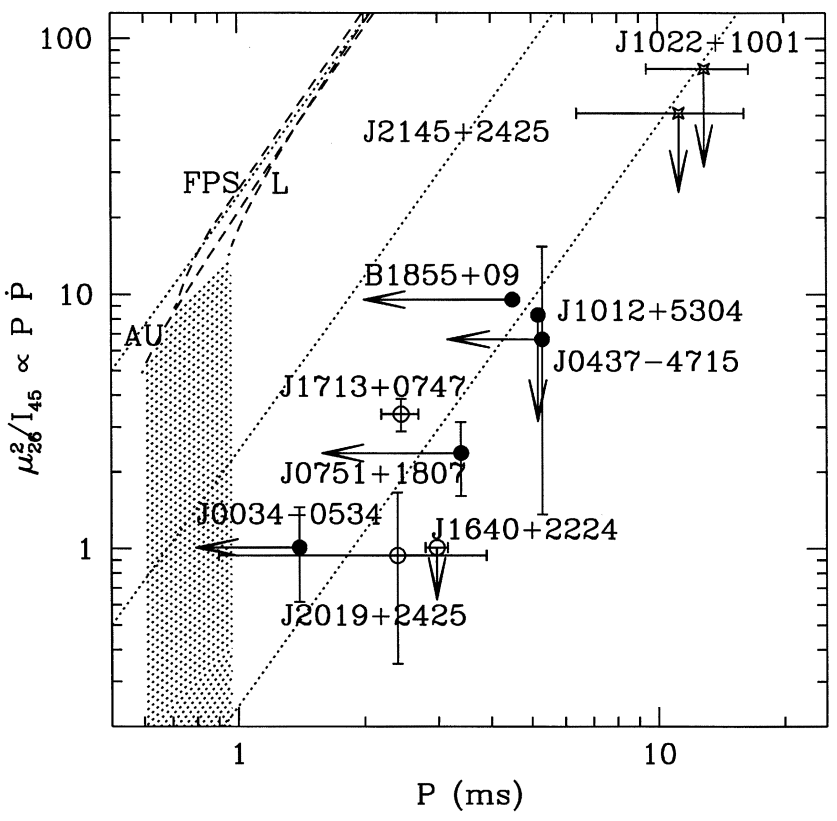

Figure 14. Initial spin periods for millisecond pulsars. We show here the inferred limits on the initial spin periods for the neutron stars discussed in the text. The three parallel dotted lines indicate the spin-up lines (8) for $\dot{M} / \dot{M}_{\text {Edd }}$ $=1,0.1$ and 0.01 respectively, and canonical neutron star values of $M=1.4$ $\mathrm{M}_{\odot}$ and $R=10 \mathrm{~km}$. The heavy dashed lines indicate the Eddington rate spinup lines for different equations of state taken from Cook et al. (1994), and spanning the range from hard $(\mathrm{L})$ to soft $(\mathrm{AU})$. The shaded region represents the case for maximal spin-up covered by the range of equations of state (i.e. they result from the spin-up of a maximum static mass model). Many of the pulsar magnetic fields are uncertain because of the Shklovskii effect on $\dot{P}$. The derivation of the limits on $P_{0}$ is discussed in the text. The open circles describe systems with orbital periods $>50 \mathrm{~d}$ while the filled circles describe those with periods $<50 \mathrm{~d}$. The two starred systems are the spiral-in systems containing carbon core companions.

In equation (7) we neglect $P_{0}$ to obtain a lower limit on $t_{\mathrm{D}}$. However, if we assume that the pulsar did begin the millisecond pulsar stage with $P_{0} \ll P$, then we may also obtain an upper limit on $t_{\mathrm{D}}$ by using the maximum allowed cooling age and minimum $t_{P}$. For most of the pulsars here this is not an interesting limit, but for $\mathrm{J} 1012+5307$ we find $t_{\mathrm{D}}<0.2 \mathrm{Gyr}$. Thus we have an alternative explanation for this pulsar's anomalous parameters. Either this pulsar was born spinning close to its current period or its magnetic field decays on a time-scale $\sim 10^{8}$ yr.

\subsection{Nuclear equation of state}

In addition to constraining evolution scenarios, we note that our models offer the possibility of determining neutron star masses and hence constraining the nuclear equation of state. If we adopt the van Kerkwijk et al. gravity in Section 3.2, then the neutron star mass is $>1.7 \mathrm{M}_{\odot}$. This would rule out five of the softer equations of state listed in Cook et al. (1994). This is also interesting, because it raises the value of the minimum rotation period for a neutron star. Of the surviving models, the shortest minimum spin period is $0.47 \mathrm{~ms}$. However, good gravity measurements and good radial velocities are required to make this a robust calculation.

If one assumes $n=3$, the initial spin period of J0034-0534 can also provide a useful constraint on the harder equations of 


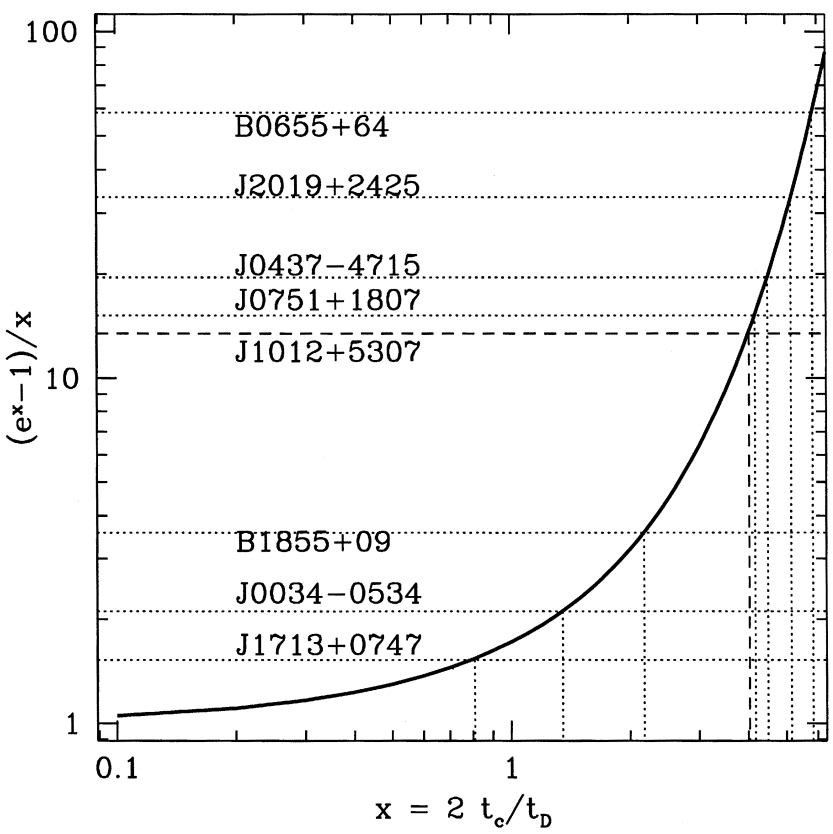

Figure 15. The heavy solid curve describes the universal function $\left(\mathrm{e}^{x}-1\right) / x$ where $x=2 t / t_{\mathrm{D}}$ and $t, t_{\mathrm{D}}$ are the cooling time and magnetic field decay time respectively. The dotted lines are the upper limits placed for the various systems as labelled. Thus, to derive a lower limit on $t_{\mathrm{D}}$, we use these limits on $x$ and the smallest allowed $t$. The dashed line shows the lower limit on $x$ derived for $\mathrm{J} 1012+5307$.

state, but this conclusion is not robust, because a braking index $n=2$ will yield an upper limit that lies above all minimum spin periods.

In conclusion, we have shown that using the white dwarf cooling ages as an independent chronometer for binary pulsars can teach us a lot about both neutron star structure and binary evolution. In particular, the determination of initial spin periods provides us with new information about the final stages of pulsar spin-up and evolution. However, we must reiterate that our results should be considered as preliminary until certain uncertainties can be conclusively addressed. In particular, except in the few cases where parallaxes are available, we are forced to use dispersion measure distances. The oft-claimed 30 per cent error is a statistical statement; individual cases may be more uncertain, as is suggested by the case of PSR B1820+02. Nevertheless, the good agreement we obtain argues that this is a reasonable method in general. Secondly, our temperature estimates are uncertain for some of the cooler members of our sample. While we have used the results of Bergeron et al. to constrain these errors, it should be possible to obtain similarly accurate atmospheres for these lower gravity cases, which will conclusively settle the issue. Finally we look forward to further observations of low-mass binary pulsars, in both radio and optical, which will undoubtedly lead to even better constraints in the future.

\section{ACKNOWLEDGMENTS}

The authors thank Marten van Kerwijk for use of results prior to publication and discussion of white dwarf observational uncertainties, and Glenn Soberman for discussions about mass transfer in binaries, as well as the referee, Frank Verbunt, whose comments contributed greatly to the clarity of this paper. This work was supported by NSF grant AST93-15455 and NASA grant NAG52756.

\section{REFERENCES}

Alberts F., Savonije G. J., Pols O. R., van den Heuvel E. P. J., 1996, Nat, 380, 676

Bailes M. et al., 1994, ApJ, 425, L41

Bailyn C., 1993, ApJ, 411, L83

Bell J. F., Bailes M., Bessell M. S., 1993, Nat, 364, 603

Bell J. F., Bailes M., Manchester R. N., Weisberg J. M., Lyne A. G., 1995, ApJ, 440, L81

Bell J. F., Bailes M., Manchester R. N., Lyne A. G., Camilo F., Sandhu J. S., 1997, MNRAS, 286, 463

Bergeron P., Wesemael F., Fontaine G., 1991, ApJ, 367, 253

Bergeron P., Saumon D., Wesemael F., 1995, ApJ, 443, 764

Bessell M. S., 1979, PASP, 91, 589

Bhattacharya D., Wijers R. A. M. J., Hartman J. W., Verbunt F., 1992, A\&A, 254,198

Bradt H. V. D., McClintock J. E., 1983, ARA\&A, 21, 13

Callanan P., Koester D., 1998, MNRAS, submitted

Callanan P. J., Charles P. A., Hassal B. J. M., Machin G., Mason K. O., Naylor T., Smale A. P., van Paradijs J., 1989, MNRAS, 238, 25P

Camilo F., 1996, in Johnston S., Walker M. A., Bailes M., eds, Proc. IAU Colloq. 160, Pulsars: Problems and Progress. Astron. Soc. Pac., San Francisco, p. 539

Camilo F., Foster R. S., Wolszczan A., 1994a, ApJ, 437, L39

Camilo F., Thorsett S. E., Kulkarni S. R., 1994b, ApJ, 421, L15

Cook G. B., Shapiro S. L., Teukolsky S. A., 1994, ApJ, 424, 823

Coté J., Pylyser E. H. P., 1989, A\&A, 218, 131

Danziger I. J., Baade D., Della Valle M., 1993, A\&A, 276, 382

Foster R. S., Wolszczan A., Camilo F., 1993, ApJ, 410, L91

Foster R. S., Cadwell B. J., Wolszczan A., Anderson S. B., 1995, ApJ, 454, 826

Ghosh P., 1995, J. Astrophys., 16, 289

Gunn J. E., Ostriker J. P., 1970, ApJ, 160, 979

Hansen B. M. S., Phinney E. S., 1997, MNRAS, 291, 569

Iben I., Tutukov A. V., 1986, ApJ, 311, 742

Johnston S. et al., 1993, Nat, 361, 613

Jones A. W., Lyne A. G., 1988, MNRAS, 232, 473

Joss P. C., Rappaport S., Lewis W., 1987, ApJ, 319, 180

Kaspi V. M., Taylor J. H., Ryba M. F., 1994, ApJ, 428, 713

Koester D., Chanmugam G., Reimers D., 1992, ApJ, 395, L107

Kulkarni S. R., 1986, ApJ, 306, L85

Kulkarni S. R., Narayan R., 1988, ApJ, 335, 755

Kulkarni S. R., Djorgovski S., Klemola A. R., 1991, ApJ, 367, 221

Lorimer D. R., Lyne A. G., Festin L., Nicastro L., 1995, Nat, 376, 393

Lundgren S. C., Zepka A. F., Cordes J. M., 1995, ApJ, 453, 419

Lundgren S. C., Cordes J. M., Foster R. S., Wolszczan A., Camilo F., 1996a, ApJ, 458, L33

Lundgren S. C., Foster R. S., Camilo F., 1996b, in Johnston S., Walker M. A., Bailes M., eds, Proc. IAU Colloq. 160, Pulsars: Problems and Progress. Astron. Soc. Pac., San Francisco, p. 497

Lyne A. G., 1996, in Johnston S., Walker M. A., Bailes M., eds, Proc. IAU Colloq. 160, Pulsars: Problems and Progress. Astron. Soc. Pac., San Francisco, p. 73

Lyne A. G., Lorimer D. R., 1994, Nat, 369, 127

Narayan R., Ostriker J. P., 1990, ApJ, 352, 222

Neckel T., Klare G., 1980, A\&AS, 42, 251

Nicastro L., Lyne A. G., Lorimer D. R., Harrison P. A., Bailes M., Skidmore B. D., 1995, MNRAS, 273, L68

Nice D. J., Taylor J. H., Fruchter A. S., 1993, ApJ, 402, L49

Pelletier C., Fontaine G., Wesemael F., Michaud G., Wegner G., 1986, ApJ, 307,242

Phinney E. S., Kulkarni S. R., 1994, ARA\&A, 32, 591

Pylyser E. H. P., Savonije G. J., 1988, A\&A, 191, 57 
Rappaport S., Podsiadlowski P., Joss P. C., Di Stefano R., Han Z., 1995, ApJ, 273, 731

Refsdal S., Weigart A., 1971, A\&A, 13, 367

Reid I. N., 1996, AJ, 111, 2000

Sandhu J. S., Bailes M., Manchester R. N., Navarro J., Kulkarni S. R., Anderson S. B., 1997, ApJ, 478, L95

Savage B. D., Mathis J. S., 1979, ARA\&A, 17, 73

Shklovskii I., 1970, SvA, 13, 562

Smarr L. L., Blandford R. D., 1976, ApJ, 207, 574

Taylor J. H., Cordes J. M., 1993, ApJ, 411, 674
Taylor J. H., Dewey R. J., 1988, ApJ, 332, 720

van den Heuvel E. P. J., 1995, JA\&A, 16, 255

van Kerkwijk M. H., Kulkarni S. R., 1995, ApJ, 454, L141

van Kerkwijk M. H., Bergeron P., Kulkarni S. R., 1996, ApJ, 467, L89

Verbunt F., 1993, ARA\&A, 31, 93

Verbunt F., Wijers R. A. M. J., Burm H. M. G., 1990, A\&A, 234, 195

Webbink R. F., 1975, MNRAS, 171, 555

Wood M. A., 1992, ApJ, 386, 539

This paper has been typeset from a $T_{E} X / L^{A} T_{E} X$ file prepared by the author. 